\title{
Modeling Sea Ice
}

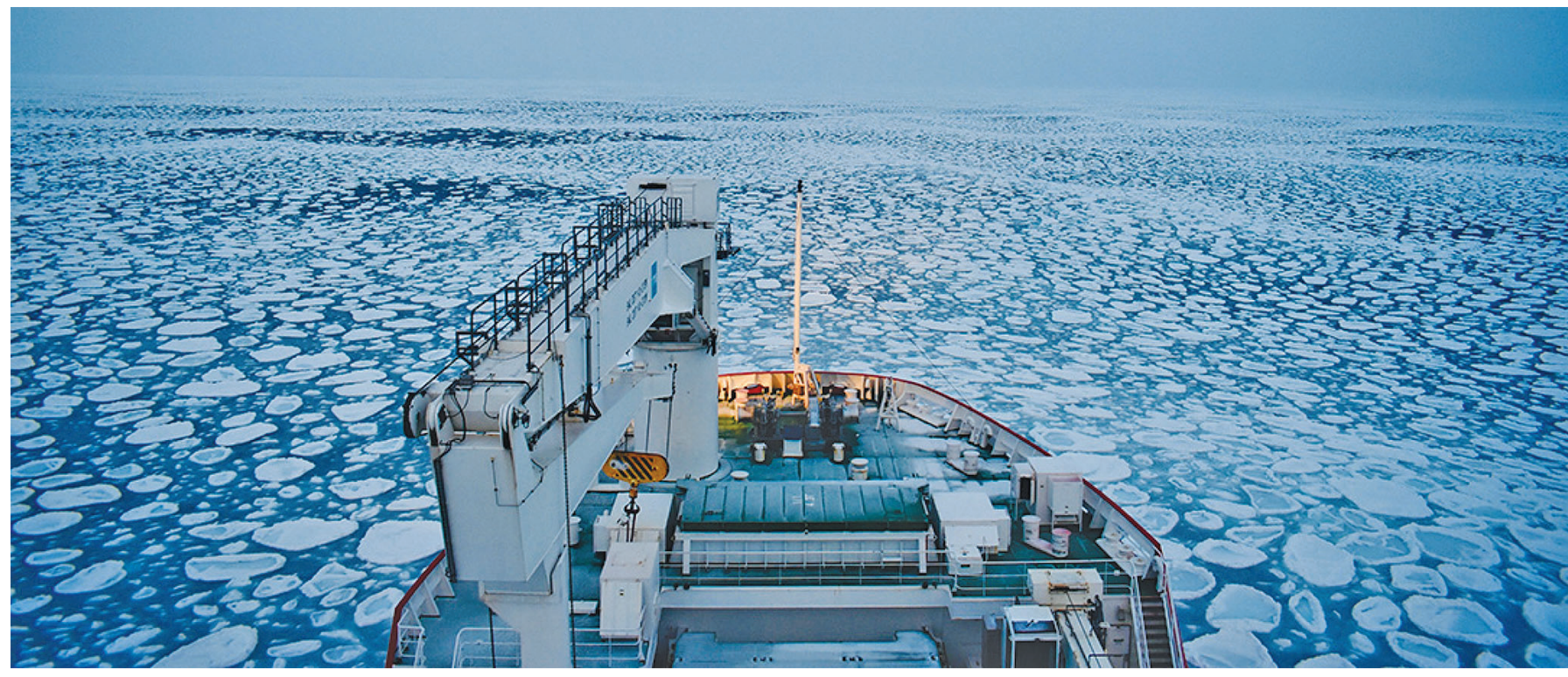

Kenneth M. Golden, Luke G. Bennetts, Elena Cherkaev, Ian Eisenman, Daniel Feltham, Christopher Horvat, Elizabeth Hunke, Christopher Jones, Donald K. Perovich, Pedro Ponte-Castañeda, Courtenay Strong, Deborah Sulsky, and Andrew J. Wells

Kenneth M. Golden is a Distinguished Professor of Mathematics at the University of Utah. His email address is golden@math . utah . edu.

Luke G. Bennetts is an associate professor of applied mathematics at the University of Adelaide. His email address is 1uke. bennetts@ade 1aide.edu. au. Elena Cherkaev is a professor of mathematics at the University of Utah. Her email address is el ena@math. utah. edu.

Ian Eisenman is an associate professor of climate, atmospheric science, and physical oceanography at the Scripps Institution of Oceanography at the University of California San Diego. His email address is ei senman@ucsd.edu.

Daniel Feltham is a professor of climate physics at the University of Reading. His email address is d. 1 . fe1 thamareading. ac.uk.

Christopher Horvat is a NOAA Climate and Global Change Postdoctoral Fellow at the Institute at Brown for Environment and Society at Brown University. His email address is christopher_horvat@brown.edu.

Elizabeth Hunke is a deputy group leader, T-3 fluid dynamics and solid mechanics group at the Los Alamos National Laboratory. Her email address is eclare (1)an1.gov.

Christopher Jones is a Bill Guthridge Distinguished Professor of Mathematics at the University of North Carolina, Chapel Hill. His email address is ckrtj Qunc.edu.

Donald K. Perovich is a professor of engineering at the Thayer School of Engineering at Dartmouth College. His email address is donald.k.perovich adartmouth.edu.

Pedro Ponte-Castañeda is a Raymond S. Markowitz Faculty Fellow and professor of mechanical engineering and applied mechanics and of mathematics at the University of Pennsylvania. His email address is ponte@seas. upenn. edu. Courtenay Strong is an associate professor of atmospheric sciences at the University of Utah. His email address is court. strong@utah. edu.

Deborah Sulsky is a professor of mathematics and statistics and of mechanical engineering at the University of New Mexico. Her email address is sulsky amath.unm.edu.

Andrew J. Wells is an associate professor of physical climate science at the University of Oxford. His email address is Andrew.We11s@physics.ox.ac.uk. Communicated by Notices Associate Editor Reza Malek-Madani.

For permission to reprint this article, please contact:

reprint-permission@ams.org. 


\section{Introduction}

Overview. Over the past two decades, Arctic sea ice has experienced precipitous declines in extent and thickness, ushering in a new narrative for the polar marine environment. This loss of sea ice is perhaps one of the most visible large-scale changes on Earth's surface connected to planetary warming, with significant implications for the Arctic region and beyond. The response of Antarctic sea ice to climate change has been more complicated and less well understood. Advancing our ability to analyze, model, and predict the behavior of sea ice is critical to improving projections of climate change and the response of polar ecosystems, and in meeting the challenges of increased human activities in the Arctic. Over the past decade or so, research on modeling sea ice and its role in Earth's climate system has blossomed, with fundamental contributions from many areas of applied and computational mathematics.

Here we report on significant recent advances in sea ice modeling. We give a fast-paced account of a broad range of mathematical ideas, and key theoretical issues in the physics of sea ice, on scales from millimeters to thousands of kilometers. Modeling sea ice-a complex multiscale medium-presents formidable challenges. The mathematics discussed here, while developed for sea ice, often has broader applicability and provides insights into the analysis and modeling of other multiscale materials and systems.

Earth's sea ice packs in a changing climate. The sea ice covers of the polar oceans are a critical component of the global climate system. They are vast in areal extent, covering millions of square kilometers, but are only a thin veneer of ice a couple of meters thick. Sea ice serves as both an indicator of change and as an amplifier of change. Consider the Arctic sea ice cover. The amplification of global warming long predicted by models has come to pass. Observations show that the Arctic is warming at twice the rate of the rest of the planet. Satellite observations from the past four decades show decreasing ice extent in every month of the year, with the greatest losses occurring in September, at the end of the melt season. Indeed, there has been interest in predictions of when we might first see substantially ice-free summers. The sea ice cover has also undergone a fundamental shift from older, thicker, more resilient perennial ice to primarily younger, thinner, less resilient seasonal ice. This is clear evidence of a warming climate.

Sea ice can also act as an amplifier of climate change through ice-albedo feedback. Snow covered sea ice is an excellent reflector of sunlight. As the ice retreats, it uncovers the highly absorbing ocean. More solar radiation is then absorbed, resulting in more loss of ice and more absorbed

DOI: https://doi.org/10.1090/noti2171 sunlight creating a positive feedback loop. The end result is less sea ice and more planetary heating. Sea ice loss also influences the exchange of heat and moisture between the atmosphere and ocean, further impacting the climate.

As we look toward the Southern Ocean and the Antarctic sea ice pack, we find a different geographical context within which to consider a more complex response to planetary warming. The Arctic is comprised of an ocean encircled by warming landmasses with declining spring and summer snow covers and reduced albedo. The Antarctic is a continent surrounded by ocean, geographically isolated from the rest of the world but connected through atmospheric and oceanic pathways. With sea ice growth unconstrained by surrounding continents, we see high variability in year to year ice extent, with strong influence from changes in wind and current patterns.

Much of the Arctic sea ice pack still persists during summer. However, relatively little Antarctic sea ice, which forms at lower, warmer latitudes and is thinner, survives the melt season. While Arctic sea ice has seen sustained declines, Antarctic sea ice experienced over three decades of gradual increases in yearly extent, peaking in 2014, yet with some regions still registering notable declines. This period was followed by an overall decline so precipitous that three years later a record low for average coverage during the 40-year satellite era was reached [S72]. Nevertheless, the long-term trend is nearly flat. In short, the planetary warming signal has come through loud and clear in the Arctic, but has so far been more difficult to discern in the response of Antarctic sea ice [S89].

From microscale to macroscale. Viewed on almost any length scale, sea ice displays composite structure (Figure 1 ). By holding a small piece of sea ice, careful inspection reveals the brine and air inclusion microstructure on the millimeter scale as well as the centimeter scale polycrystalline microstructure. Snow on top of sea ice is a highly variable granular material with grains on the millimeter to centimeter scale. From a helicopter the ice pack can be viewed as a composite of ice floes in a sea water host, with the frozen "inclusions" ranging in size from centimeters to tens of kilometers. Systems of leads or openings in the ice are fracture patterns that can extend hundreds of kilometers. In late spring the Arctic sea ice surface is a composite of ice and small ponds which grow and coalesce to form kilometer scale, connected labyrinths of melt water.

In modeling and predicting the behavior of the polar sea ice covers, one must keep in mind the overall scale of the system under study. In winter, the maximum areal extent of sea ice in the Arctic is about 15 million $\mathrm{km}^{2}$, and about 18 million $\mathrm{km}^{2}$ in the Antarctic, or roughly $4000 \mathrm{~km}$ in linear extent. With grid spacings in large-scale models on the order of kilometers, or more likely, tens of kilometers, it is not realistic to account for every brine inclusion, 

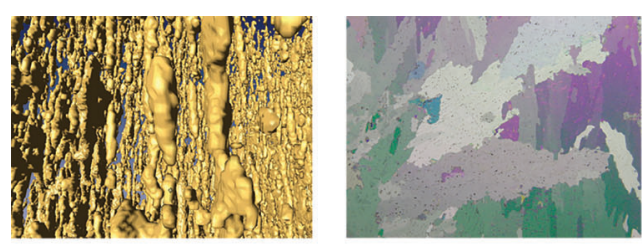

millimeters

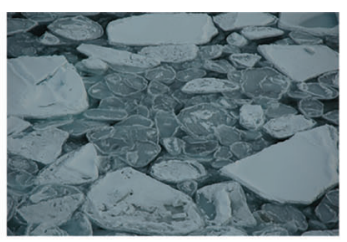

centimeters
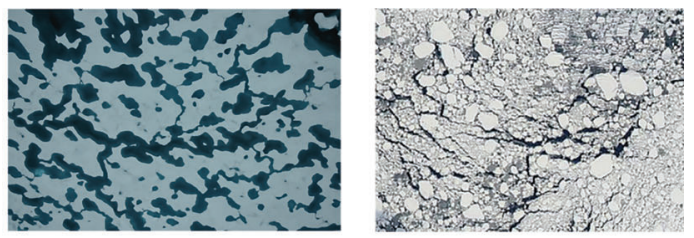

kilometers

Figure 1. Multiscale structure of sea ice. From left to right: X-ray tomography of submillimeter scale brine inclusions; centimeter scale polycrystalline structure of sea ice; centimeter to meter scale pieces of newly forming Arctic sea ice; meter to kilometer scale melt ponds on the surface of summer Arctic sea ice; the Arctic sea ice pack can be viewed from space as a two-phase granular composite. For perspective, rough image sizes from left to right are $1 \mathrm{~cm}, 5 \mathrm{~cm}, 5 \mathrm{~m}, 100 \mathrm{~m}, 100 \mathrm{~km}$.

crystal grain, or floe in the model. The scales of interest for climate studies are far greater than these "microstructural" scales. Nevertheless, these features play an outsized role in sea ice dynamic and thermodynamic behavior. One of the fundamental challenges in modeling sea ice-and a central theme in what follows-is how to rigorously account for the impact of the microscale on macroscale behavior.

Most of the modeling ideas and techniques in the following apply to sea ice around both poles. However, there are certain topics that are traditionally more relevant to either the Arctic or the Antarctic. For example, melt ponds are generally not observed on Antarctic sea ice. Grainy polycrystalline microstructures, often associated with growth under more turbulent conditions or the formation of "snow-ice" on top of flooded sea ice, have typically been of more interest in studies of Antarctic sea ice. Likewise, studies involving wave-ice interactions and pancake ice, which forms in wavy conditions, have been more focused in the Southern Ocean. However with Arctic sea ice receding, wave activity has increased, along with similar types of studies in the Arctic.

The paper is organized as follows. We begin with an introduction to the basic physics of sea ice-its dynamics and thermodynamics - that has historically served as the foundation for early modeling efforts, and still serves in this role today. Then we roughly follow the outline suggested in Figure 1, where we move up in scale, starting from the material properties of sea ice and how they depend on the brine and polycrystalline microstructures. We then consider mesoscale processes such as the formation of leads and ridges, the evolution of melt ponds and floe sizes, and wave-ice interactions. Finally we look at the macroscale-the rheology of the sea ice pack, large-scale numerical sea ice models which are key components of global climate models, the assimilation of data into these models, and low-order approximations of large-scale sea ice behavior. Along the way we will touch upon a number of areas of mathematics and theoretical physics, including homogenization of partial differential equations, theory of porous media, mechanics of materials, numerical analysis, percolation theory, stochastic processes, complex analysis, statistical mechanics, dynamical systems, and even random matrix theory.

\section{Sea Ice Dynamics and Thermodynamics}

Physical processes acting on sea ice can be divided into two categories: thermodynamic processes, which involve the transfer of heat or radiation, and dynamic processes, which move and deform the ice. Regions of the sea ice cover often include mixtures of open water, thin first-year ice, thicker multiyear ice, and thick ridges formed from ice floes breaking or colliding under convergent forcing.

A fundamental goal in sea ice modeling is to predict the evolution of the ice thickness distribution (ITD) in time and space [TRMC75, S112]. The ITD is influenced by horizontal transport, ridging and other mechanical processes, and thickness changes due to thermodynamic growth and melting. An essential aspect of sea ice thermodynamics is the variation of growth and melting rates for different ice thicknesses. Because heat conduction is proportional to the vertical temperature gradient, thin ice grows and melts more quickly than thicker ice, and is more likely to undergo mechanical deformation.

The ice thickness distribution $g(h, \mathbf{x}, t)$ gives the probability $g(h) d h$ of finding ice in the thickness range $(h, h+d h)$ at a given time $t$ and location $\mathbf{x} \in \mathbb{R}^{2}$, or the area fraction covered by ice of this thickness, with $\int_{0}^{\infty} g(h, \mathbf{x}, t) d h=1$. The ITD equation is fundamental to any sea ice model, because it integrates all of the physical processes that affect ice volume:

$$
\frac{\partial g}{\partial t}=-\nabla \cdot(g \mathbf{u})+\Psi-\frac{\partial}{\partial h}(f g)+L,
$$

with $\mathbf{u}$ the horizontal ice velocity, $\nabla=\left(\frac{\partial}{\partial x}, \frac{\partial}{\partial y}\right), f$ the rate of thermodynamic ice growth, $\Psi$ a mechanical redistribution function, and $L$ representing lateral melting. The four terms on the right describe: (1) horizontal transport in $\mathbf{x}$, (2) transport of mass of $g$ in $h$ due to ridging and other mechanical processes, (3) transport of mass of $g$ due to thermodynamic growth and melting, and (4) replacing ice with open water by lateral melt. Solving the horizontal transport and ridging equations requires $\mathbf{u}$.

Although the ITD $g(h)$ is a function of a continuous variable $h$, in practice only a few thickness categories are 
tracked, typically between 5 and 20, with more categories assigned to thinner ice to better resolve growth rates. Thermodynamic transport of the mass of $g(h)$ needs the ice growth rate $f$ in each thickness category. The ITD equation is being generalized to include distributions of thickness and floe size. In a significant recent advance, the ITD was treated as a Fokker-Planck equation for the probability density of a thickness diffusion process $h_{t}$, with $\Psi$ similar to a Boltzmann collision term [S103].

Dynamics. In order to simulate ice motion $\mathbf{u}$, sea ice models generally include equations for the momentum, a constitutive law that describes the material properties of the ice, transport, and mechanical deformation. These equations strive to represent the basic properties of ice motion: as a rigid material it resists convergent forcing, but it tends to be highly fractured and therefore diverges easily. If convergent forcing is strong enough, the ice will break and form ridges and keels, often referred to as "ridging." The deformation or rates of strain, which are spatial derivatives of the velocity components, control the amount of ridging and fracture, which in turn determines the amount of open water that is exposed to the atmosphere.

The force balance per unit area in the ice pack is given by a two-dimensional momentum equation, obtained by integrating the three-dimensional equation with respect to the thickness of the ice in the vertical direction:

$$
m \frac{\partial \mathbf{u}}{\partial t}=\nabla \cdot \sigma+\tau_{a}+\tau_{w}-C m \mathbf{k} \times \mathbf{u}-m g \nabla H,
$$

where $m$ is the combined mass of ice and snow per unit area and $\tau_{a}$ and $\tau_{w}$ are wind and ocean forces, respectively. The internal stress $\sigma$ is given by a constitutive equation describing sea ice strength and rheology. The last two terms represent Coriolis effects and sea surface slope $H$, where here $g$ is gravitational acceleration.

Ice area fraction, volume, energy, and snow volume and energy are advected horizontally. In addition, coupled to equation (2) are equations governing the transport of tracers such as melt water and biogeochemical inclusions.

Thermodynamics. Thermodynamic components of sea ice models treat the ice as a slab with energy fluxes at both surfaces,

$$
q \frac{d h}{d t}=F_{S}+F_{l}+F_{L \downarrow}+F_{L \uparrow}+(1-\alpha) I_{0} F_{s w},
$$

where $q$ is the energy per unit volume required to melt the top surface material (either snow or ice), $h$ is thickness, $F_{S}$ is the sensible heat flux, $F_{l}$ is the latent heat flux, $F_{L \downarrow}$ is the incoming longwave flux, $F_{L \uparrow}$ is the outgoing longwave flux, $F_{s w}$ is the incoming shortwave flux, $\alpha$ is the shortwave albedo, and $I_{0}$ is the fraction of absorbed shortwave flux that penetrates into the ice. A similar relation holds at the bottom of the ice. The heat equation in the vertical direction for the interior temperature $T_{i}$ inside the ice is

$$
\rho_{i} c_{i} \frac{\partial T_{i}}{\partial t}=\frac{\partial}{\partial z}\left(k_{i} \frac{\partial T_{i}}{\partial z}\right)-\frac{\partial}{\partial z}\left[I_{p e n}(z)\right],
$$

where $\rho_{i}$ is sea ice density, $c_{i}$ its specific heat, $k_{i}$ its (effective) thermal conductivity, and $I_{p e n}$ is the flux of solar radiation penetrating to depth $z$ (downward positive). Heat capacity and conductivity depend on both salinity and temperature.

Some thermodynamic models treat sea ice as a mushy layer [S122,S24], a mixture of brine and ice, with enthalpy (or temperature) and salinity as prognostic variables. The enthalpy $q$ is related to the temperature $T$ and brine volume $\phi$ by

$$
\begin{aligned}
q & =\phi q_{b r}+(1-\phi) q_{i} \\
& =\phi \rho_{w} c_{w} T+(1-\phi)\left(\rho_{i} c_{i} T-\rho_{i} L_{0}\right),
\end{aligned}
$$

where $q_{b r}$ is brine enthalpy, $q_{i}$ is ice enthalpy, $\rho_{i}$ and $c_{i}$ are density and heat capacity of ice, $\rho_{w}$ and $c_{w}$ are density and heat capacity of brine, and $L_{0}$ is the latent heat of melting pure ice.

Many processes affect sea ice thermodynamics, including interactions of long- and shortwave radiation with ice surface characteristics (snow, bare ice, melt water) and interior layers, turbulent fluxes (evaporation, latent and sensible heat fluxes, wind stress), heat fluxes and stresses from the ocean, and algal growth, which can darken the ice, decreasing the albedo.

\section{Sea Ice as a Material}

As sea ice grows from the freezing of sea water at $-1.8^{\circ} \mathrm{C}$ and a typical salinity of 35 parts per thousand (ppt), brine is entrained in the ice. The volume fraction of brine in sea ice depends on the ice temperature and salinity, as well as the age and initial growth rate of the ice. The presence of this brine is a distinctive feature of sea ice. The amount and distribution of the brine affects all aspects of sea ice including its electromagnetic, mechanical, and thermal properties [S116,S74].

The influence of the brine phase on the bulk material properties of sea ice depends strongly on temperature. In particular, in sea ice colder than, say, $-15^{\circ} \mathrm{C}$, the brine microstructure typically occupies less than $2 \%$ volume fraction, and is segregated into submillimeter scale inclusions or pores which are largely disconnected and well separated, with diminished overall influence. However, as the temperature warms toward around $-5^{\circ} \mathrm{C}$ and above, longrange order develops as the inclusions coalesce to form centimeter to meter scale connected structures, or brine channels, through which fluid can flow.

The conditions under which sea ice forms determine its crystallographic structure. The centimeter scale crystals are columnar in shape for ice grown under quiescent 
conditions, and more granular when grown in wavy or turbulent seas, which affects the material properties of sea ice.

Remote sensing and sea ice properties. One reason the material properties of sea ice are so important and why there is significant interest in understanding and modeling them, particularly sea ice electromagnetic properties, is that they largely determine what is "seen" by remote sensing platforms focused on the sea ice pack. Observations from satellite-based remote sensing provide large-scale information on the spatial variability and temporal evolution of the polar sea ice covers. The relationships between the observed electromagnetic signatures and the physical state of the ice provide large-scale information on ice conditions. At the heart of these relationships is the mathematics and physics of electromagnetic wave interactions with a complex multiscale composite material that has rough surfaces and significant internal variability [S33].

There is an array of satellite instrumentation including passive microwave, radar, visible and near-infrared, thermal infrared sensors, high resolution photography, and radar and lidar altimeters. The electromagnetic signatures are converted by algorithms into information on sea ice concentration and extent, ice age, ice motion, ice surface conditions, and ice thickness [S52]. Underlying these algorithms are various electromagnetic inverse problems and inverse scattering theories [S32]. The resulting data sets are a critical contribution to observing and understanding the changing polar sea ice cover.

Homogenization refers broadly to a circle of ideas in applied mathematics and the physics and engineering of materials, where the goal is to find the effective, bulk, or homogenized properties of a composite or inhomogeneous medium. Sea ice is a polycrystalline composite of pure ice with brine, air, and solid salt inclusions. We first consider homogenization for two-phase composites and then for polycrystalline media.

We briefly describe the analytic continuation method (ACM) in homogenization theory. This approach was originally developed to study the effective properties of twophase composite materials, such as electrical and thermal conductivity, complex permittivity, magnetic permeability, diffusivity, and elasticity, which can all be similarly formulated [S9,S62,S29,S63]. The method has been used to obtain forward bounds on the homogenized coefficients given partial information on the microstructure, such as the volume fractions of the constituents, and extended to multiphase media using techniques of several complex variables [S30,S28]. This approach has also been successful in addressing the inverse homogenization problem of obtaining information on the microstructural parameters from bulk property measurements [S58,S16,S13,S63].

Motivated by sea ice remote sensing and the physics of electromagnetic waves interacting with sea ice, there were numerous developments in the 1990s in the ACM and its application to sea ice [Gol09]. Most of this work was focused on the brine microstructure. More recently, again motivated by sea ice processes, there have been several advances in extending the ACM to larger-scale problems. These include homogenization for polycrystalline materials [GLCG15], advection diffusion processes involving incompressible velocity fields $[\mathrm{S} 4, \mathrm{~S} 66]$, such as thermal transport through sea ice enhanced by brine convection [S51], and ocean surface wave propagation through the sea ice pack treated as a two-phase composite of ice floes and sea water [S84].

To describe the ACM and the principal features that carry over to other systems, we formulate the method in the electromagnetic case for the complex permittivity, although equivalent formulations could be given for the other properties mentioned above. We consider a twophase locally isotropic composite, $\epsilon(\mathbf{x})=\epsilon_{1} \chi_{1}(\mathbf{x})+\epsilon_{2} \chi_{2}(\mathbf{x})$, where $\epsilon_{j}$ is the complex permittivity of brine or ice for $j=1,2$, respectively, and $\chi_{j}$ is the characteristic function equaling 1 for medium $j$ at $\mathbf{x}$, and 0 otherwise, with $\chi_{2}=1-\chi_{1}$. The local parameter $\epsilon(\mathbf{x})$ is a stationary random field with $\Omega$ the set of realizations of the random medium and underlying probability measure $P$ compatible with stationarity [Gol09, S29].

When the wavelength is much larger than the microstructural scale, the problem can be formulated with the quasistatic Maxwell equations, $\nabla \times \mathrm{E}=0, \nabla \cdot \mathbf{D}=0$, where $\mathbf{E}(\mathbf{x})$ and $\mathbf{D}(\mathbf{x})$ are stationary electric and displacement fields with $\mathbf{D}(\mathbf{x})=\epsilon(\mathbf{x}) \mathbf{E}(\mathbf{x})$. We assume $\langle\mathbf{E}\rangle=\mathbf{e}_{k}$, where $\langle\cdot\rangle$ denotes ensemble averaging over $\Omega$ or spatial averaging over all of $\mathbb{R}^{d}$, and $\mathbf{e}_{k}$ is a unit vector in the $k$ th direction. The effective complex permittivity tensor $\epsilon^{*}$ is defined by $\langle\mathbf{D}\rangle=\epsilon^{*}\langle\mathbf{E}\rangle$. Let $\epsilon^{*}=\epsilon_{k k}^{*}=\left\langle\epsilon \mathbf{E} \cdot \mathbf{e}_{k}\right\rangle$. Due to homogeneity, $\epsilon^{*}\left(a \epsilon_{1}, a \epsilon_{2}\right)=a \epsilon^{*}\left(\epsilon_{1}, \epsilon_{2}\right), \epsilon^{*}$ depends on $h=\epsilon_{1} / \epsilon_{2}$ and we define $m(h)=\epsilon^{*} / \epsilon_{2}$, which is a Herglotz function that maps the upper half $h$-plane to the upper half $m$-plane, and is analytic off $(-\infty, 0]$. The key is to obtain the resolvent representation

$$
\mathbf{E}=s\left(s \mathbf{I}+\boldsymbol{\Gamma} \chi_{1}\right)^{-1} \mathbf{e}_{k} .
$$

Here $\Gamma=\nabla\left(-\Delta^{-1}\right) \nabla \cdot$ is a projection from $L^{2}(\Omega, P)$ onto the Hilbert space of curl-free random fields, and $\Delta^{-1}$ is convolution with the free space Green's function for the Laplacian $\Delta=\nabla^{2}$.

Consider $F(s)=1-m(h), s=1 /(1-h)$, which is analytic off $[0,1]$ in the s-plane. Then $(6)$ yields a Stieltjes integral representation for $\epsilon^{*}$,

$$
F(s)=\int_{0}^{1} \frac{d \mu(z)}{s-z},
$$

where $\mu(d z)=\left\langle\chi_{1} Q(d z) \mathbf{e}_{k} \cdot \mathbf{e}_{k}\right\rangle$ is a positive spectral measure on $[0,1]$ and $Q(d z)$ is the (unique) projection-valued 
measure associated with the bounded, self-adjoint operator $\Gamma \chi_{1}$.

Equation (7) is based on the spectral theorem for the resolvent of $\boldsymbol{\Gamma} \chi_{1}$. This representation separates the component parameters in $s$ from the geometrical information in $\mu$. The geometry enters via the moments $\mu_{n}=$ $\int_{0}^{1} z^{n} d \mu(z)=\left\langle\chi_{1}\left[\left(\boldsymbol{\Gamma} \chi_{1}\right)^{n} \mathbf{e}_{k}\right] \cdot \mathbf{e}_{k}\right\rangle, n=0,1,2, \ldots$. The mass $\mu_{0}$ is $\left\langle\chi_{1} \mathbf{e}_{k} \cdot \mathbf{e}_{k}\right\rangle=\left\langle\chi_{1}\right\rangle=\phi$, where $\phi$ is the brine volume fraction. The $(n+1)$-point correlation function of the medium determines $\mu_{n}$.

The integral representation (7) yields forward bounds on the effective parameters of composites, given partial information on the microgeometry via the $\mu_{n}[\mathrm{~S} 63]$, and on $\epsilon^{*}$ for sea ice in particular [Gol09]. The integral representation can also be used to obtain inverse bounds, allowing one to use data about the electromagnetic response of a composite, for example, to bound its structural parameters, such as the volume fraction of each of the components [S58,S16,S13,S63,S38,S69].

Computing the spectral measure $\mu$ directly for a composite microstructure involves discretizing an image of the composite into a square lattice filled with 1's and 0's corresponding to the two phases, as in Figure 2. Then $\Gamma \chi_{1}$, which depends on the geometry via $\chi_{1}$, becomes a matrix. The spectral measure may be calculated from the eigenvalues and eigenvectors [S65].

(a)

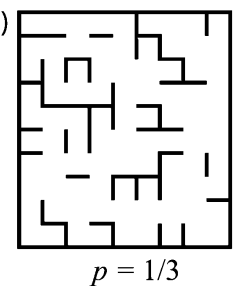

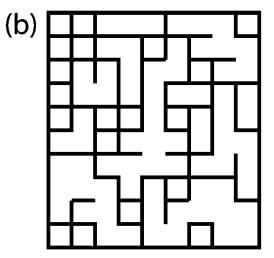

$p=2 / 3$

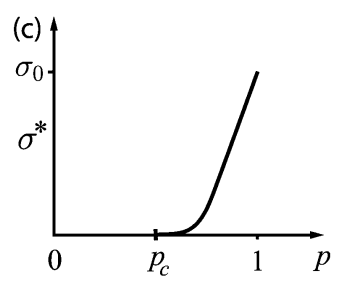

Figure 2. The two-dimensional square lattice below its percolation threshold of $p_{c}=1 / 2$ in (a) and above it in (b). A schematic graph of the effective conductivity is shown in (c).

Percolation. The connectivity of the brine phase in sea ice is a principal determinant of its electromagnetic and fluid transport properties. For example, fluid convection in sea ice, which can occur when the brine phase is sufficiently connected to form channels, plays an important role in thermal transport in sea ice as well as in nutrient replenishment processes for microbial communities living inside the brine inclusions. On larger scales, the connectivity of melt ponds on the surface of Arctic sea ice helps determine drainage patterns which can impact sea ice albedo. In ocean surface wave propagation through the two-phase composite of ice floes in a sea water host, the connectivity of the water phase significantly influences the homogenized mechanical properties and wave propagation characteristics of the ice pack.
The percolation model has been widely used to formulate and address questions involving connectivity in complex systems such as porous media and composite materials [Gol09]. In its simplest form, one considers the $d$ dimensional integer lattice $\mathbb{Z}^{d}$, and the square or cubic network of bonds joining nearest neighbor lattice sites. We assign to each bond a conductivity $\sigma_{0}>0$ with probability $p$, meaning it is open (black), or a conductivity 0 with probability $1-p$, meaning it is closed. Two examples of bond configurations are shown in Figure 2, with $p=1 / 3$ in (a) and $p=2 / 3$ in (b). Groups of connected open bonds are called open clusters. In this model there is a critical probability $p_{c}, 0<p_{c}<1$, called the percolation threshold, which is the smallest $p$ for which an infinite open cluster exists. For the two-dimensional bond lattice $p_{c}=1 / 2$.

The effective conductivity $\sigma^{*}(p)$ of the lattice, or random resistor network (RRN) defined via Kirchoff's laws, vanishes for $p<p_{c}$ as shown in Figure 2(c), since there are no conducting pathways. For $p>p_{c}, \sigma^{*}(p)>0$, and near $p_{c}, \sigma^{*}(p) \sim \sigma_{0}\left(p-p_{c}\right)^{t}, p \rightarrow p_{c}^{+}$, where $t$ is the conductivity critical exponent, with $1 \leq t \leq 2$ in $d=2,3$, and numerical values $t \approx 1.3$ in $d=2$ and $t \approx 2.0$ in $d=3$. Now consider a random pipe network with effective fluid permeability $\Pi(p)$ and critical behavior $\Pi(p) \sim \Pi_{0}\left(p-p_{c}\right)^{e}$, where $\Pi_{0}$ depends on pipe radius and $e$ is the permeability exponent, with $e=t$ for lattices. Both $t$ and $e$ are believed to be universal-depending only on dimension and not on the lattice. Continuum models, like the Swiss cheese model, can exhibit nonuniversal behavior with exponents different from the lattice case and $e \neq t$.

Columnar sea ice, which forms under quiescent conditions, has a percolation threshold of $\phi_{c} \approx 5 \%$ brine volume fraction. For a typical bulk salinity of $5 \mathrm{ppt}$, this corresponds to a critical temperature of $T_{c} \approx-5^{\circ} \mathrm{C}$, known as the rule of fives [GAL98]. In modeling brine geometry to predict the low value of $\phi_{c}$, we observe an excluded volume effect: the inclusions lie on the boundaries of pure ice platelets, not randomly distributed throughout their host. Sea ice is similar to compressed powders of polymer spheres with smaller conducting particles in the interstices, used in radar absorbing, stealthy aircraft coatings. Connectivity of the conducting phase in compressed powders can be achieved with low volume fractions of the particles. A continuum theory of compressed powders adapted to sea ice predicts the $5 \%$ threshold-the on-off switch for fluid flow in sea ice [GAL98]. Brine inclusion sizes are lognormally distributed, so that lattice theory holds. Universal critical exponents $e=t=2$ give predictions for $\Pi(\phi)$ and $\sigma^{*}(\phi)$ that agree closely with measurements [S34].

The spectral measure $\mu$ depends on the microstructure of the composite, and phase connectedness in particular. For the RRN with the microgeometry determined by independent weighted coin flips, $\Gamma \chi_{1}$ becomes a matrix with 
random coefficients via $\chi_{1}$, which equals 1 on open bonds. The statistics of the eigenvalues and eigenvectors of $\boldsymbol{\Gamma} \chi_{1}$ for discretizations of sea ice structures and the RRN were studied. It was found that as long-range order and connectivity develop with $p \longrightarrow p_{c}^{-}$, the spectral and localization statistics undergo a percolation-driven Anderson transition [S64]. This behavior directly parallels the quantum theory of the metal/insulator transition and other localization phenomena in wave physics [S37], and connects sea ice modeling to random matrix theory [S21]. The eigenvalue spacing distribution, for example, transitions from an uncorrelated Poissonian to a repulsive, universal Wigner-Dyson distribution for the Gaussian Orthogonal Ensemble [S64].

Polycrystalline structure. Sea ice is a polycrystalline composite-a conglomeration of centimeter scale individual crystals, whose structure depends on how the ice was grown [S116,S74]. For congelation ice frozen under calm conditions, the crystals are vertically elongated columns, and each crystal itself is a composite of pure ice platelets separating "layers" of brine inclusions. The orientation of each crystal is determined by the direction that the $c$-axis points, which is perpendicular to the platelets. Horizontal thin sections of columnar sea ice viewed under crosspolarized light are shown in Figures 1 and 3. Shown on the right in Figure 3 is a thin section of granular ice, likely grown under turbulent conditions, or perhaps formed as snow-ice from flooding of the surface snow layer and subsequent freezing.

The $c$-axes of columnar ice typically lie within the horizontal plane, yet are randomly oriented within the plane, unless there is a prevalent ocean current direction during growth. In this case the $c$-axes tend to align with the current $[S 117, S 54]$, as shown in Figure 3. The orientations of the crystals in granular ice tend to be statistically isotropic, as shown on the right in Figure 3, where different colors indicate different crystal orientations. The columnar ice on the left, on the other hand, has $c$-axes that are closely correlated.

The polycrystalline structure of sea ice can impact its electromagnetic and mechanical properties, as well as how

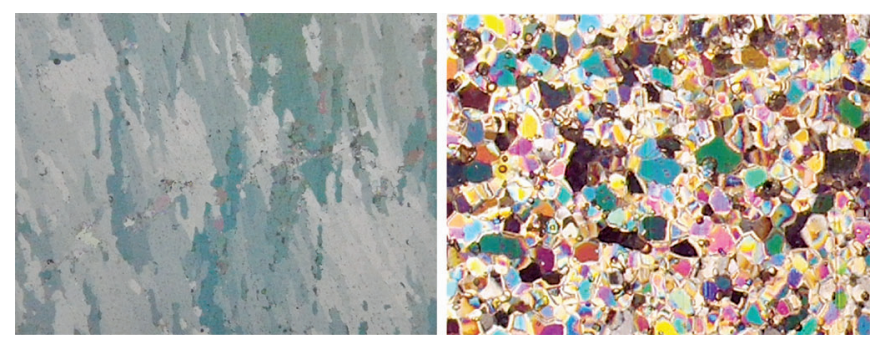

Figure 3. Cross-polarized images of columnar sea ice from the Ross Sea on the left, and granular ice from the Bellingshausen Sea on the right.
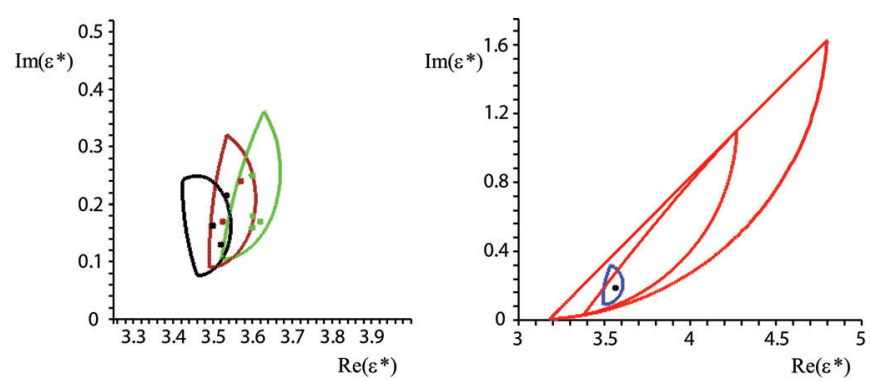

Figure 4. Polycrystalline bounds [GLCG15] on the complex permittivity of sea ice (left) together with measurements [S2]. Comparison of a polycrystalline bound (blue) with the two component bounds (right) shows a dramatic improvement over the classical results as the new bounds include additional information about single crystal orientations (notice different scales on the axes).

fluids and nutrients flow through the ice. Recent work, for example, shows that granular ice has a higher percolation threshold for fluid flow than columnar ice, with $\phi_{c} \approx 10 \%$, which has implications for modeling microbial communities and physical processes [S35]. Determining ice type using remote sensing techniques is thus of particular interest. Early studies show that aligned columnar ice gives different radar returns and observed permittivities, depending on the electric field polarization, affecting measurements of ice thickness [S31].

In extending the analytic continuation approach beyond two-phase composites, a Stieltjes integral representation and bounds were obtained for $\epsilon^{*}$ of polycrystalline composites in general, and sea ice in particular, considered as a three-dimensional, transversely isotropic or uniaxial polycrystalline composite material [GLCG15]. The forward bounds on the components of $\epsilon^{*}$ use information about the complex permittivity tensor $\boldsymbol{\varepsilon}$ of the individual crystals and the mean crystal orientation. In Figure 4 (left) they are in good agreement with measurements [S2], and (right) they are compared with the classic two-component bounds. The inverse bounds [GLCG15, S16] for the mean orientation are obtained from measurements of $\epsilon^{*}$, and lay the groundwork for determining ice type using remote sensing techniques.

The mathematical framework for analysis of the electromagnetic transport properties of random, uniaxial polycrystalline media [GLCG15] is analogous to that for twophase random media. In a polycrystalline material, crystals of varying size, shape, and orientation have the same complex permittivity tensor $\boldsymbol{\varepsilon}$ with different values along different crystal axes. Crystal orientation is given by a rotation matrix $\mathbf{B}$.

For transversely isotropic or uniaxial polycrystalline media, the permittivity along one of the crystal axes has the value $\epsilon_{1}$, while the permittivities along all the other crystal axes have the value $\epsilon_{2}$, so that $\varepsilon=\operatorname{diag}\left(\epsilon_{1}, \epsilon_{2}, \epsilon_{2}\right)$. The local 
permittivity tensor of such media is given by

$$
\epsilon(\mathbf{x}, \omega)=\mathbf{B}^{T}(\mathbf{x}, \omega) \varepsilon \mathbf{B}(\mathbf{x}, \omega),
$$

where $\mathbf{B}(\mathbf{x}, \omega)$ is a random rotation matrix. The effective complex permittivity tensor $\epsilon^{*}$ is defined as above, and has components $\epsilon_{j k}^{*}=\mathbf{e}_{j} \cdot \epsilon^{*} \mathbf{e}_{k}=\left\langle\mathbf{e}_{j} \cdot \mathbf{B}^{-1} \varepsilon \mathbf{B E}\right\rangle$, where $\langle\mathbf{E}\rangle=$ $\mathbf{e}_{k}$. Introducing $h=\epsilon_{1} / \epsilon_{2}, s=1 /(1-h)$, and matrices $\mathbf{C}=\mathbf{e}_{1}\left(\mathbf{e}_{1}\right)^{T}$ and $\mathbf{R}=\mathbf{B}^{T} \mathbf{C B}$, we can write components of a tensor function $F_{j k}(s)$ as $F_{j k}(s)=1-\epsilon_{j k}^{*} / \epsilon_{2}=\left\langle s^{-1} \mathbf{e}_{j} \cdot \mathbf{R E}\right\rangle$. In analogy with the two-component case, we derive the resolvent representation

$$
\mathbf{E}=s(s \mathbf{I}+\mathbf{\Gamma R})^{-1} \mathbf{e}_{k},
$$

which leads to an integral representation for $F_{j k}(s)$ similar to (7) where the positive-definite measure $\left\{\mu_{j k}\right\}$ is the spectral measure of the self-adjoint operator $\Gamma R=\nabla(-\Delta)^{-1} \nabla$. R.

The inverse bounds estimate the mean crystal orientation from $\epsilon^{*}$ data, and show a significant difference in the reconstructed mean orientations for columnar and granular ice. This provides a foundation for distinguishing ice types using electromagnetic measurements. The Stieltjes integral representation can be generalized to elastic $[S 48, S 71]$ and viscoelastic composites [S10,S15,S70], and to viscoelastic polycrystalline materials [S14].

The polycrystalline structure of sea ice also strongly influences its rheological behavior. Although there can be significant variations depending on the age and depth of the ice, at microscopic scales, sea ice floes can be considered to be made of solid polycrystalline ice with brine inclusions embedded in the hexagonal closed packed (HCP) ice grains. The dominant structure at this scale consists of columnar grains that exhibit a pronounced texture with $c$-axes of the HCP single crystals in the horizontal plane and with random orientations in this plane. As the HCP ice crystals exhibit highly anisotropic viscoplastic behavior, with "easy" glide on basal planes and "hard" slip on nonbasal systems, this specific texture strongly influences the macroscopic response at these length scales. Similarly, the elongated intragrain brine inclusions also strongly affect the rheological response of sea ice.

In [DC19] and [S76,S92] a homogenization model has been developed that accounts for the viscoplastic anisotropy of the crystal grains in sea ice, the average shape, volume fraction $\phi$, and orientation of the brine inclusions, as well as the crystallographic texture and average grain shape and orientation. One crucial finding from these models is that the viscoplastic response of intact sea ice exhibits a nonlinear dilatational response, which is due to the accommodation of overall volumetric strain by concomitant changes in the porosity. In addition, the hydrostatic nonlinear viscosity of sea ice depends strongly on the porosity and average pore shape-tending to decrease with increasing porosity and aspect ratio.

Mushy layer theory and brine channels. As thinner firstyear ice becomes more prevalent in the Arctic Ocean, replacing thicker, fresher, multiyear ice, we are led to consider sea ice with higher porosity and greater susceptibility to interstitial fluid flow. We then focus on brine transport over the scale of the ice thickness (i.e., from centimeter to meter scales) which is effectively characterized using continuum models of flow in reactive porous media [S47] The resulting fluid dynamics drives convective brine rejection during winter ice growth, which controls surface buoyancy fluxes across the polar oceans. Such flows also provide chemical and nutrient transport for biogeochemical systems [S111]. Because the porous sea ice is reactive, salt transport induces porosity variations that impact material properties, such as the permeability variations that impact formation of surface melt ponds [S75]. These dynamics are also of intrinsic mathematical interest, featuring freeboundary evolution, nonlinear dynamics, and pattern formation in a multiscale continuum system that undergoes a transient evolution through the relevant dynamical phase space. We refer the reader to [WHP19] and [S47,S123] for further details and references.

A widely applied continuum modeling approach treats sea ice as a mushy layer: a two-phase, two-component reactive porous material [S122,S24]. We consider phaseweighted dynamics over representative volume elements containing many ice crystals and liquid brine pores, with porosity $\phi$, temperature $T$, and phase-weighted salinity $\bar{S}=S_{l} \phi+S_{S}(1-\phi)$ for liquid salinity $S_{l}$ and solid salinity $S_{S} \approx 0$. The pore scale microstructure is assumed to adjust sufficiently rapidly to maintain local thermodynamic equilibrium, with local phase changes modifying the liquid salinity so that the mixture lies at the freezing temperature $T=T_{f}\left(S_{l}\right)$. Conservation of energy, salt, momentum, and mass result in the following system of coupled PDEs:

$$
\begin{gathered}
\overline{\rho c_{p}} \frac{\partial T}{\partial t}+\rho_{l} c_{l} \mathbf{u} \cdot \nabla T=\nabla \cdot\left(k^{*} \nabla T\right)-\rho_{s} L \frac{\partial \phi}{\partial t}, \\
\frac{\partial}{\partial t}\left[S_{l} \phi\right]+\mathbf{u} \cdot \nabla S_{l}=\nabla \cdot\left(D^{*} \nabla S_{l}\right), \\
\frac{\partial}{\partial t}\left[\rho_{l} \phi+\rho_{s}(1-\phi)\right]+\nabla \cdot\left(\rho_{l} \mathbf{u}\right)=0, \\
\frac{\nu}{\Pi} \mathbf{u}=-\nabla p+\rho \mathbf{g},
\end{gathered}
$$

where $\overline{\rho c_{p}}$ is the homogenized heat capacity (at constant pressure), $\rho_{l} c_{l}$ is the heat capacity of the liquid, $k^{*}$ is the effective thermal conductivity, $\rho_{s}$ and $\rho_{l}$ are the solid and liquid densities, $L$ is the latent heat, $D^{*}(\phi)$ is the homogenized salt diffusivity, $v$ is the dynamic viscosity, and $\mathbf{g}$ is gravitational acceleration. The momentum equation (13) uses Darcy's law for flow in a porous medium, where the averaged velocity field $\mathbf{u}$ depends on the fluid permeability 
$\Pi(\phi)$, the pressure gradient, and buoyancy forces depending on fluid density $\rho \approx \rho_{l}\left(1-\beta S_{l}\right)$ with haline coefficient $\beta$.

For $\mathbf{u}=0$, (10)-(11) can be used with appropriate boundary conditions to solve a Stefan problem for a diffusively growing ice layer with vertical variation of the porosity $\phi$. However, for natural sea ice growth an unstable density gradient arises in the pore fluid, and a convective instability can break the horizontal symmetry giving rise to brine channels [S122]. The convective cells are modified by a flow-focusing feedback, where downwelling brine dissolves the ice matrix, increasing porosity and permeability. The nonlinear development focuses downflow into narrow high porosity brine channels that eventually become solid free, with wider regions of upwelling and lower porosity in between [S122]. The resulting brine channels evolve in space and time as the ice grows, as shown in experiments reviewed in [WHP19], and in numerical solutions of the nonlinear system (10)-(13) illustrated in Figure 5. The question then arises as to what controls the wavelength of this pattern, and the resulting brine channel spacing.

Linear stability analyses predict convective onset when the mushy-layer Rayleigh number $R=\rho_{l} g \beta \Delta S \Pi_{0} h / \kappa \nu$ exceeds a critical value [S122], which occurs for large enough mushy-layer thickness $h$. Here $\Delta S$ is a characteristic salinity difference, $\Pi_{0}$ a representative, permeability value, and $\kappa$ the thermal diffusivity. Weakly nonlinear analyses elucidate the pattern formation, and potential for oscillatory modes of instability [S1,S122]. However, the channel spacing coarsens over time, and fully developed brine channels are solid free, which represents a significant departure from the background state. This requires a different approach that accounts for the nonlinearity. One such approach uses enthalpy method simulations, where the narrow brine channel widths promote the effectiveness of Adaptive Mesh Refinement [PMWK20]. Asymptotic models have also been developed to describe brine channel flow [S87], exploiting the slenderness of channel width $a$ versus the mushy-layer thickness $(a / h \ll 1)$. The asymptotically reduced channel description has been coupled to numerical models of the remaining mush [S120] and used in semianalytic models [S79].

For steady growth with a periodic array of channels with imposed wavelength $\lambda$, the salt fluxes from the mushy layer vary with the channel spacing. The competition between neighboring channels results in a saddle-node bifurcation with flow shutting down as $\lambda$ is decreased [S79,S118]. This is consistent with experiments of mushy-layer growth with lateral confinement [S126]. For unrestricted growth in wider domains, it has been posed [S118] that the emergent wavelength in this nonlinear dissipative system evolves to optimize the salt flux (and corresponding flux of potential energy). This predicts that the channel spacing scales
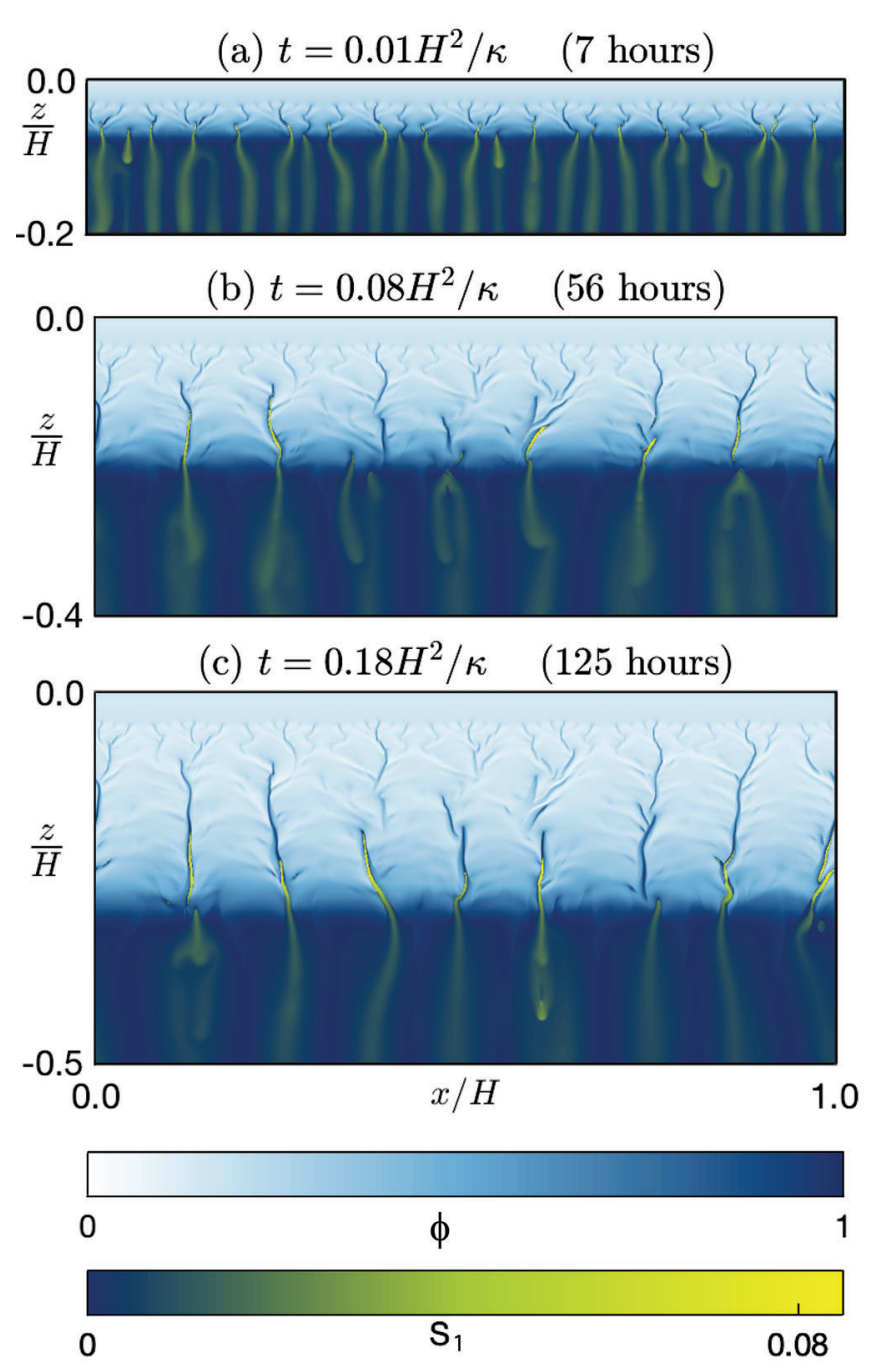

Figure 5. Evolution of a convecting mushy layer with porosity $\phi$ and dimensionless liquid-region salinity

$S_{1}=\left(S_{l}-S_{o}\right) /\left(S_{E}-S_{o}\right)$, where $S_{o}$ is ocean salinity and $S_{E}$ eutectic salinity. Simulations use the enthalpy method in [PMWK20] for sea ice growth in a 2-D Hele-Shaw cell of depth $H$.

proportionally to the depth of convective cells, and yields a solute flux consistent with transient ice growth in lab experiments [S119]. More detailed tests of the transient evolution are awaited, but this approach has motivated parameterizations of fluid transport for brine fluxes and biogeochemical systems [S123].

\section{Mesoscale Processes}

Formation of leads and ridges. The Arctic Ocean is surrounded by land and each fall sea water freezes up to the land boundaries and fills the Arctic basin with ice that lasts through the winter. Near-shore ice melts in the spring and summer but (currently) ice remains in the high Arctic year round. Motion of the ice is driven primarily by wind and ocean currents, with wind being the dominant force. 
As ice forms from frozen sea water, it insulates the relatively warmer ocean $\left(-2^{\circ} \mathrm{C}\right)$ from the colder atmosphere $\left(-20^{\circ} \mathrm{C}\right)$. When the atmosphere is cold, water initially freezes rapidly, but as the insulating layer of ice grows, the freezing rate slows, as a basic Stefan model shows. At thermodynamic equilibrium, the ice would be about $1.5 \mathrm{~m}$ thick; however ice motion and deformation alter ice thickness. Leads are areas of open water formed when currents or winds pull or shear ice apart, and in winter form long, narrow openings, meters to hundreds of meters wide that can stretch hundreds of kilometers or more in length [S22]. Leads occupy $1-2 \%$ of the ice area but account for $70 \%$ of the ocean-air heat flux [S56]. (Heat flux through ice is 2$5 \mathrm{Wm}^{-2}$ compared with $300-500 \mathrm{Wm}^{-2}$ through leads.) Leads are of fundamental importance to Earth's heat budget, ice production, and navigation.

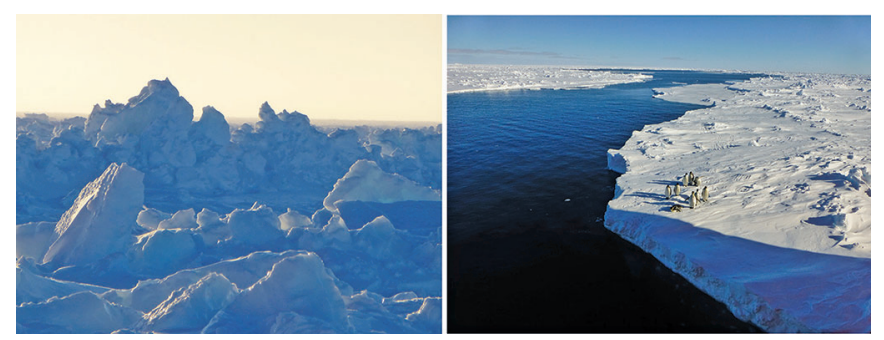

Figure 6. Left: Ridged Antarctic sea ice. Right: A wide lead in Antarctic sea ice.

Within a lead, rapid refreezing of the open water creates thin ice. The crushing of the thinner lead ice during convergent or shear flow piles blocks of ice onto the surface to form ridge sails or forces the blocks under the surface to form ridge keels. These prominent features of the Arctic ice pack can reach roughly $30 \mathrm{~m}$ high. It is estimated that half of the total Arctic ice volume is in ridged ice [S112]. Bonds formed between the ice blocks due to freezing, and refreezing of the part under the water surface, turn sea ice ridges into robust ice features. Ridge formation is a mechanism that increases the volume of ice per unit area. Both leads and pressure ridges are usually narrow, long, localized features, often referred to as linear kinematic features [S53].

Ridges have variable properties and shapes [S102,S97] which change throughout the season [S55], but data has been difficult to obtain. Data on block dimensions, keel and sail width and area, mechanical properties, and internal structure such as degree of consolidation or porosityand the relationships between these properties-are lacking. To fill the gaps, individual ridge formation has been studied analytically using beam theory [S73,S19], numerically using DEM, in ice tank tests [S109], and through remote sensing via satellites and upward-looking sonar mounted on submarines.
A realistic representation of leads and ridges in sea ice models could drastically increase the fidelity in large-scale general circulation models. The opening and closing of open water areas in leads affects ice production and ice mass balance, vertical heat fluxes between the ocean and atmosphere, and upper ocean salinity since brine is rejected as sea water freezes. Leads have an albedo significantly lower than sea ice and thus increase the local absorption of solar energy. Ridge sails and keels change the atmosphereice and the ice-ocean drag forces. Modeling corresponding effective drag coefficients involves complex homogenization problems. Ridges appear not only in high Arctic regions, but also in areas where human activities are more prevalent. On the floe scale, leads are areas where land- and ice-dwelling animals feed, and where sea life or submarines can surface. The design of off-shore structures and ice-going vessels needs to account for possible loads imparted by ridging ice.

Floe size distribution. Viewed from a helicopter or satellite, the sea ice cover is a composite material-a mosaic of individual pieces, known as floes. Each individual floe may be identified with two geometric parameters: its thickness and its "size," where size is a metric related to its horizontal extent. Whereas ice thicknesses vary from centimeters to meters, floe sizes vary from pancakes centimeters across to swaths of connected ice in winter, reaching over hundreds of kilometers, on the scale of the entire Arctic basin.

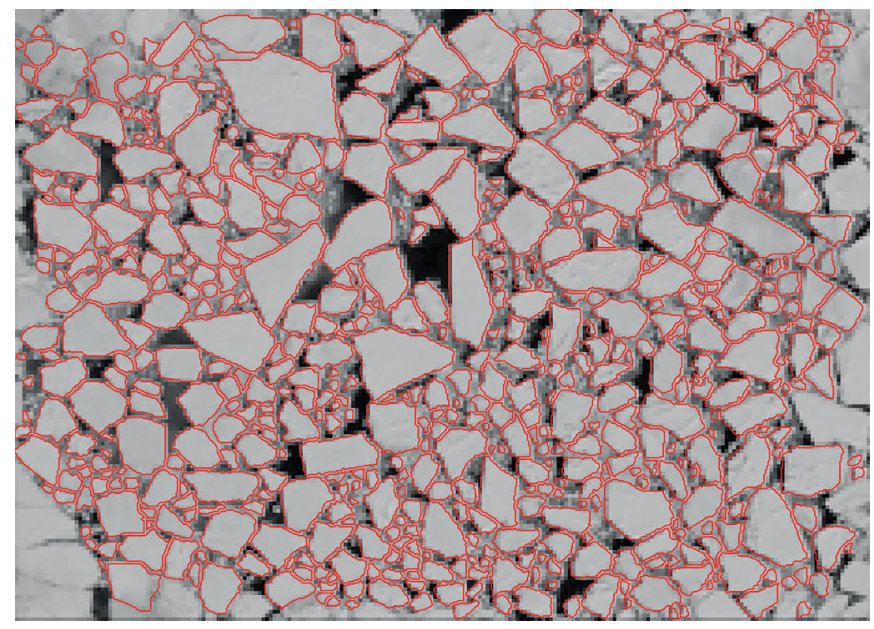

Figure 7. Segmentation of a video image via thresholding gives floe boundaries (red) in the Antarctic marginal ice zone [S104].

The significance of the shape and scale of sea ice floes was recognized in a modeling context in [S83], where a variety of different approaches to compiling statistical information about floe sizes was described. The "mean caliper diameter" $2 r_{C}$ was defined as the average distance between two parallel lines that touch a floe's boundary but do not 
intersect it. Though floes have irregular shapes, the relation $A=\pi r_{C}^{2}$, where $A$ is floe area, was found to be accurate within $10 \%$, and therefore recent modeling efforts define a floe's size through the effective radius of an equivalent-area circular disc [HT15], $r \equiv \sqrt{A / \pi}$.

Consider an area $\mathcal{A}$ containing $\mathcal{N}$ floes with areas $A_{i}$ and radii $r_{i}$. An area-based floe size distribution (FSD) $f(r)$ is defined analogously to the ice thickness distribution $g(h)$ as

$$
f(r) d r=\frac{1}{\mathcal{A}} \sum_{i \in R_{i}} A_{i},
$$

where $R_{i}=\left\{i: r_{i} \in[r, r+d r)\right\}$. The FSD is therefore an area-weighted probability distribution function of floe size defined at any scale.

The FSD encodes information about the number of floes per unit area at each size $N(r)=f(r) / \pi r^{2}$, and the perimeter of floes per unit area at each size $P(r)=2 f(r) / r$. Upon visual inspection, ice pack geometry exhibits selfsimilar scale-invariant behavior. In [S83] and nearly all subsequent observational studies, power law behavior for these distributions was adopted, such as $N(r) \propto r^{-\alpha}$ for $r \in\left[r_{0}, \infty\right)$. The power-law exponent $\alpha$ is directly analogous to a fractal dimension under the assumption that some scale-invariant process generates the FSD, for example sequential fragmentation [S104]. Note, however, that observed power law coefficients can be produced by many different scale-dependent processes [S45], and the analogy is not necessarily appropriate in all cases. Constraints on $\alpha$ are imposed by physical constraints: finite floe area and finite or infinite floe perimeter.

The "power law hypothesis" has been supported in some observational studies, with different $\alpha$ observed in different size regimes [S106,S104], though the general validity of this hypothesis has seldom been tested. Studies of $f(r)$ without straight lines in log-log space are often interpreted as "double power law distributions" [S106]. The first Arctic-wide assessment of the FSD using satellite altimetry showed limited support for power-law tail behavior [S44]. Recent meta-analyses of FSD observations $[\mathrm{S} 39, \mathrm{~S} 94]$ also reveal a wide range of reported values of $\alpha$ ( $\sim$ between 0 and 4 ) and a lack of consistent scaleinvariant behavior. Still, there is clear utility in parametric descriptions of the FSD.

Viewing the sea ice pack as a granular composite material, floe size is analogous to grain size in traditional continuum mechanics [S107]. Sea ice models that use a continuum viscous-plastic rheology inherit a latent subgrid scale floe size distribution [Hib79, S105]. A critical influence on the FSD is coupling to ocean surface waves. Waves directly alter the FSD, but can then also indirectly influence ice thickness or concentration, with fractured floes melting more rapidly than larger ones. In 2009 an expedition left a region of compact multiyear ice before a storm
[S3]. Returning three days later, the ice was heavily fragmented, and had mostly melted. The impact of floe size on melting has been codified [S125,S46,S80,S6], finding that wave-induced fracture changes synoptic sea ice patterns by increasing susceptibility to melting.

Most theoretical works have posited models for powerlaw FSD behavior [S23,S104,S121,S124]. In analogy to the fragmentation of brittle media, power law distributions were hypothesized using a "renormalization group" method [S104]. The fragmentation process assumes a floe of perimeter $\mathcal{P}$ fractures into $m$ equally-sized floes of perimeter $\mathcal{P} / m$ with probability $p$. The sequential application of this process yields closed-form expressions for the number-size distribution and exponent $\alpha$, which require $0<\alpha<2$. However in many observational studies, $\alpha$ does not lie within that range [S39,S94,S44]. Such scale-invariant fragmentation may exist in the interior Arctic, where sea ice is brittle, solid, and strongly constrained by geometry-but in marginal seas and in summer, sea ice is closer to free drift and it fragments either because of thermal processes or waves. A meta-analysis [S39] explored whether the wide range of observed FSDs was better fit by a Pareto distribution,

$$
N(x) \sim x^{-1-\alpha} e^{c(1-\alpha) / x},
$$

where $x=r / \bar{r}$ is floe size scaled by the mean observed size $\bar{r}$, and $c$ and $\alpha$ are unknown. Such a functional form is the solution to a discrete-time Langevin equation, with a prediction for $\alpha$ [S39] as the representation of the dual processes of floe breakup and growth.

A prognostic theory of FSD evolution built from floefloe interactions was developed in [HT15]. The FSD $f$ evolves in time according to a partial differential equation with stochastic terms,

$$
\frac{D f}{D t}=\mathcal{L}_{T}+\mathcal{L}_{M}+\mathcal{L}_{W}
$$

where $\mathcal{L}_{T}$ includes thermodynamic effects, like floe melting, growth, and welding; $\mathcal{L}_{M}$ includes mechanical effects such as rafting and ridge formation; $\mathcal{L}_{W}$ includes wave-ice interactions. Each pair of individual processes and their relationship to observations was evaluated in [S46]. Observed multiscale distributions are produced as an emergent feature [S80], implemented in climate models, and compare favorably to floe statistics from altimetry in the marginal ice zone [S44].

Because modern large scale climate models can't resolve floes, further refinements in coupled model grids may necessitate new approaches to simulating sea ice. One is hybrid continuum-discrete-element models that parameterize the FSD where floes are small relative to the grid scale, and resolve floes when they are large.

Surface wave propagation through the sea ice pack. Ocean surface waves carry huge stores of energy across the 
ocean, and can propagate hundreds of kilometers into the sea ice pack. They create a highly dynamic region known as the marginal ice zone (MIZ), in which they break up large floes into smaller floes, and promote formation of pancake ice (see opening graphic). Thus, waves influence $f(r)$ in the MIZ, giving the ice cover dynamic and thermodynamic properties distinct from the inner pack.

With sea ice weakening and retreating in response to rising temperatures, new regions of the ocean surface are opening for wave generation [S101], waves are propagating farther into the pack, the ice is becoming more susceptible to wave impacts [S3], and waves are reaching Antarctic ice shelves with potentially catastrophic consequences [S57]. This is driving integration of wave-ice processes into the next generation of global sea ice models. Accurate predictions of wave propagation through the ice pack are essential to empower predictions of the extent and properties of the MIZ, where the primary quantity of interest is the attenuation coefficient $\gamma(\omega)$, which is the rate of exponential decay of wave energy with distance travelled through the MIZ, and depends on wave frequency $\omega$ as well as the properties of the ice cover. In general, $\gamma$ increases with increasing $\omega$, so that short waves are filtered out close to the ice edge, and longer waves propagate farther into the pack.

The first standard approach to model wave propagation through the ice pack is based on multiple scattering theory, analogous to light scattering in the sky. It is used in the regime where wavelengths are comparable to floe sizes. The scattering model was initially developed in the 1970s and 80s, alongside pioneering Arctic field experiments [S113]. A wave is scattered by each floe it encounters, due to the impedance mismatch between open water and ice covered water, with floes modeled as floating elastic plates, so they flex in response to waves. The scattered wave field interacts with surrounding floes, which rescatter it, and so on, to create a multiple scattering wave field, which, under some simplifying assumptions, can be written as

$$
\eta=\eta_{\text {inc }}+\sum_{p, m} b_{m, p} \mathrm{H}_{m}\left(k r_{p}\right) \mathrm{e}^{\mathrm{i} m \theta_{p}},
$$

where $\eta$ is the ocean surface elevation, $\eta_{\text {inc }}$ is the incident wave elevation, $p \in \mathcal{F}$ is the set of floes, $\mathrm{H}_{m}$ is the first-kind Hankel function of order $m \in \mathbb{Z}, k(\omega)$ is the open-water wave number, $\left(r_{p}, \theta_{p}\right)$ is the polar coordinate of a point on the ocean surface from floe $p, b_{m, p}$ are the scatteredwave amplitudes to be calculated, and harmonic time dependence is implicitly assumed. Each individual floe has to be resolved in the model, making direct computations extremely expensive over the hundreds of kilometers that waves propagate, and extracting the attenuation coefficient from (17) is challenging. The original model was 2D (one depth dimension and one propagation dimension), and used crude approximations for scattering by an individual floe and multiple scattering by many floes.

Mathematicians have given considerable attention to the scattering model since the 1990s, developing new methods for efficient computation of attenuation rates. Some 30-40 years after the model was initially proposed, we are at the point where efficient techniques are available to solve the scattering problem for an elastic floe of arbitrary shape in three dimensions [S59,S7], and solve the $3 \mathrm{D}$ multiple scattering problem for a full sea of floes. The work in [MSB16] is a milestone in this field. It was the first to predict propagation of directional wave spectra into an ice pack consisting of order 10,000 floes with a realistic FSD. The final breakthrough was development of a slabclustering method, where the ice cover is divided into computationally manageable groups (slabs), and a recursive algorithm combines the groups.

The second standard approach is to model the ice pack as a continuum with effective properties, based on homogenization theory for composite media, including an effective viscous dissipation. It is valid in the long-wavelength regime, where wavelengths are much greater than floe sizes-for example, in pancake ice conditions. The continuum model leads to a dispersion relation in wave number $\kappa$ and frequency $\omega$,

$$
(1+Q(\kappa, \omega)) g \kappa=\omega^{2}
$$

in which $Q$ encodes the effective properties, and from which the attenuation coefficient is extracted as $\gamma=$ $2 \operatorname{Im}(\kappa)$. An early model of this type [S115] treated the ice pack as a viscous fluid floating on the ocean surface. The full dispersion relation is derived in [S50] along with asymptotics for the wave number in certain limits.

Over the past decade, there has been a resurgence in continuum models, motivated by substantially improved measurements of waves in the MIZ [S60,S100], which have predominantly been in the long-wavelength regime. A viscous-elastic continuum model [S114], which extends the viscous layer models, has received considerable attention. Effective parameters in these models have generally been fitted to experimental data, with varying success [S61]. However, in [S84] a resolvent for the strain field like equation (6) was used to obtain a Stieltjes integral and bounds for the effective complex viscoelasticity of a twophase composite of ice floes and sea water. Given the viscoelasticities of the constituents, the floe area fraction and geometry, the theory predicts effective behavior that agrees with observations.

Laboratory experiments have revealed nonlinear processes that affect propagation characteristics [S8]. Most attention has been on overwash phenomena, where waves break over the surface of floes, generating turbulent bores that propagate across the floe [S67]. A hyperbolic PDE 
system for floe overwash has been proposed, using linear theory for water around the floe, and the nonlinear shallow water equations for the overwash [S91], which gives excellent agreement with laboratory experiments. See [S93] for a recent review of progress in wave-ice interactions.

Melt ponds on Arctic sea ice. A distinctive, significant feature of the summer Arctic sea ice cover is the extensive ponding, as shown in Figure 8 . The water produced by melting snow and ice collects on the surface forming melt ponds. Ponds play a critical role in the evolution of the sea ice cover. As surface reservoirs for freshwater, they can affect the thermohaline stratification of the upper ocean. The greatest impact of melt ponds is on the albedo of the sea ice cover. Melt ponds are darker than bare ice, with albedos that can range from 0.1 to 0.4 compared to 0.6 to 0.9 for bare and snow-covered ice. The spatial coverage and albedo of ponds are highly variable in space and time. The area covered by ponds follows a seasonal cycle and also fluctuates from day to day. Melt ponds are the most intractable component of determining the summer albedo of Arctic sea ice. To understand the evolution of summer ice albedo, you must understand the evolution of melt ponds.

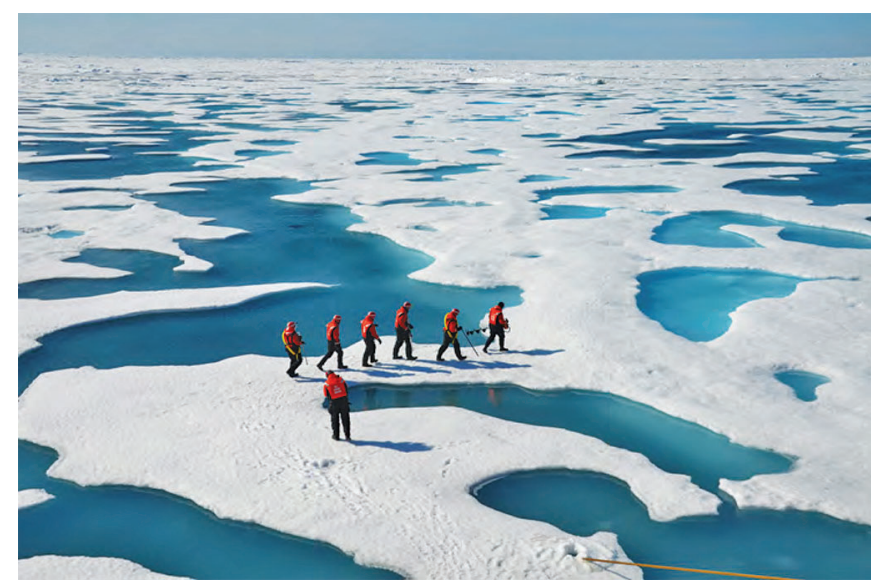

Figure 8. Melting Arctic sea ice.

The first model of melt ponds derived from physical principles was introduced in [S99]. This was a onedimensional model that treated the sea ice as a mushy layer [S24] with or without a snow cover, subject to a surface energy balance and ocean boundary conditions. The energy balance equation in the sea ice takes the form of nonlinear reactive diffusion equations in heat and salinity with a body source term of absorbed radiation. As the internal liquid fraction changes, latent heat is released/absorbed and the brine salinity alters. Radiation fluxes are calculated to account for reflection, absorption, and scattering. Surface melting generates a pond on top of the ice, which reduces the albedo. Vertical drainage of meltwater into the ocean is calculated using Darcy's law for flow in a porous medium.

While the melt pond model in [S99] describes the vertical evolution of melt ponds, the quantity of greater general interest to climate modellers is the melt pond area fraction, since this largely determines the albedo of the sea ice surface. A cellular automata model was introduced in [S88] that split a sea ice floe into a checkerboard, with each cell of the grid comprising a column of snow, ice, and melt pond of varying heights. The one-dimensional model in [S99] was applied in each cell, with melt water moving to adjacent cells by flowing downhill at a rate calculated from Darcy's law. The model simulates pond evolution, both vertically and horizontally, over various types of sea ice surfaces, with results in agreement with observations.

The above models helped provide a physical understanding and predictive capability for melt pond evolution on the scale of individual ponds and sea ice floes, however, they were (and are) too complex to be directly incorporated into climate models. To this aim, parameterizations of melt ponds were developed in $[S 25, S 26]$ which have subsequently been incorporated into climate sea ice models. Climate models do not represent the topography of the sea ice surface, which is a significant limitation for modeling melt ponds since their evolution is to a large extent determined by meltwater flowing downhill. Climate sea ice models do, however, contain a sea ice thickness distribution function $g(h, \mathbf{x}, t)$. At each time step, the melt water in a grid cell is calculated and "poured" over the ice surface, with $g(h)$ serving as an adequate proxy for surface height. The water covers the lowest ice first and then higher/thicker categories. This procedure determines the area fraction of the melt ponds and their depth on each ice thickness category.

The surface meltwater distribution model was combined with models accounting for flushing of meltwater through the ice (as in [S99]), conservative advection of meltwater between grid cells, and run-off of meltwater through cracks, to create the melt pond parameterization [S25,S26]. Despite its simplicity, atmosphere-forced simulations of sea ice using this parameterization produce pond fractions in accordance with observations, and demonstrate how important it is to account for melt ponds in long-term sea ice predictions [FSFH12]. The impact of melt ponds on sea ice mass balance is significant and mostly driven by their impact on surface albedo. Moreover, it was found that knowing pond area fraction enables skillful predictions of the summer minimum sea ice extent up to three months in advance [SFFT14].

In another line of inquiry, melt pond geometry has recently been investigated. It was found from area-perimeter data that (see the photos in Figures 8 and 9) as the ponds grow and coalesce, they display a transition in fractal 
geometry, evolving from simple shapes into complex, selfsimilar regions whose boundaries behave like space-filling curves [S42]. The fractal dimension of the boundary curves transitions from 1 to about 2 around a critical area of 100 $\mathrm{m}^{2}$. These findings constrain the geometry of melt pond evolution, provide a check on numerical simulations, and help quantify the mechanisms of pond growth impacted by the area-perimeter relationship, such as lateral heat transfer.

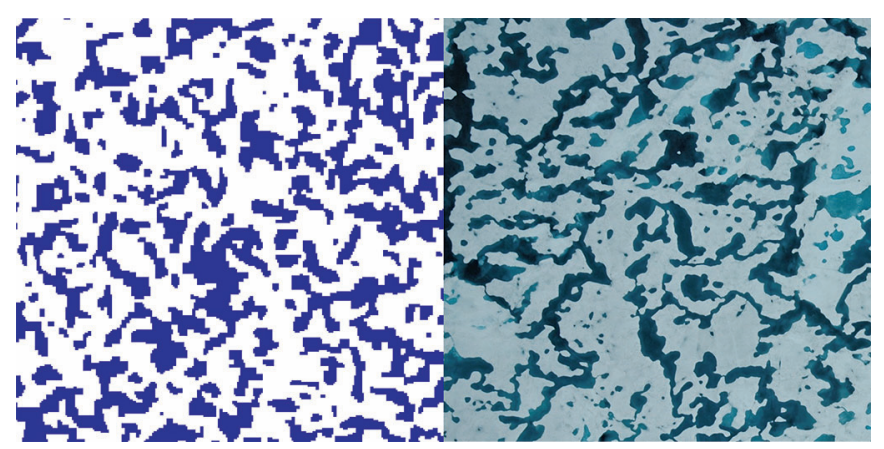

Figure 9. Ising model simulation on the left; melt pond photo on the right.

Continuum percolation models of melt pond evolution that display the observed fractal transition have been developed. In the random surface model [S11], a melt pond boundary is the intersection of a surface representing the snow topography with a horizontal plane representing the water level. As the plane rises the ponds grow and coalesce. Snow topography data are used to generate random Fourier surfaces with realistic ponds, and a framework to analyze how pond geometry depends on topography. In the void model [S77], disks of varying size which represent ice are placed randomly on the plane, with the voids between them representing the ponds. Data on pond sizes and correlations are incorporated into the model, yielding observed behavior.

Finally, the Ising model, originally developed 100 years ago to explain ferromagnetism, has been adapted to predict melt pond geometry [MSSG19, S36]. We envision a square lattice of surface patches or pixels of melt water or ice that interact only with their nearest neighbors. The lattice spacing, as determined by snow topography data, is the only measured parameter input into the model. Minimization of the melt pond Ising Hamiltonian via Glauber spin flip dynamics drives the system from an initially random state toward realistic pond configurations (see Figure 9), which are local energy minima, or metastable states. The model captures the essential mechanism of pattern formation of melt ponds, with predictions that agree very closely with observed pond size scaling and fractal transition.

\section{Large Scale Sea Ice Models}

Sea ice rheology. A given sea ice floe may be a relatively uniform ice sheet, as might be typical for relatively young ice, but is more often a refrozen patchwork of smaller pieces formed at various times which has undergone inplane and out-of-plane failure. In-plane failure results in cracks, along which ice floes grind in lateral motion. Outof-plane failure results first in break up of the ice into blocks under bending failure, followed by the pile up of blocks into the air and ocean to form pressure ridges and keels, respectively. Given this spatial and temporal heterogeneity, formulation of an effective relationship between the sea ice stress tensor $\sigma_{i j}$ and the large-scale deformation of the ice cover, i.e., the sea ice rheology, is a challenging problem that remains an active research topic.

While early models of sea ice rheology made assumptions such as treating sea ice as a viscous fluid, the study of rheology was transformed during the Arctic Ice Dynamics Joint Experiment (AIDJEX) of the 1970s, which led to the AIDJEX sea ice model [S18]. The chief advance of the AIDJEX model-rheologically-was to treat sea ice as an elastic-plastic material. The strongest argument for a plastic model is that local events such as ridging and lead formation occur sporadically and irreversibly, as though a critical stress state in the ice had been reached.

The case for an elastic (subcritical) response can be argued physically in a dense pack, where thick floes are wedged together so that as subyield stresses are applied there can be only elastic deformations. In [Hib79], the plastic approach was adopted but the elastic subyield behavior was replaced with viscous behavior, which avoids tracking an evolving unload configuration from which to measure strain. Hibler's version of this rheology, the Viscous-Plastic (VP) rheology, continues to be in widespread use today.

In [S18] and [Hib79], and most subsequent large-scale modeling efforts, sea ice is considered to be isotropic. While sea ice can exhibit rheological anisotropy in the horizontal plane under various conditions, the assumption of isotropy, on average, over sufficiently large scales was deemed reasonable [Hib79]. In recent years this assumption has been questioned with observations of largescale oriented lead patterns in Arctic sea ice and associated anisotropic models [Fel08]. However, isotropy was and remains a useful simplifying feature for modeling. Under this assumption, the plastic yield surface reduces to a yield curve in the plane of the principal stresses $\sigma_{1}$ and $\sigma_{2}$ or, equivalently, through the stress invariants $\sigma_{I} \equiv$ $\frac{1}{2}\left(\sigma_{1}+\sigma_{2}\right)=$ negative pressure and $\sigma_{I I} \equiv \frac{1}{2}\left(-\sigma_{1}+\sigma_{2}\right)=$ maximum shear stress. The yield criterion is written as

$$
\mathcal{F}\left(\sigma_{I}, \sigma_{I I} ; \text { scalars }\right)=0,
$$

where $\mathcal{F}$ is the yield function, defining a family of yield 
curves in the $\left(\sigma_{I}, \sigma_{I I}\right)$-plane as the scalar properties of the ice vary. For isotropic materials, $\mathcal{F}$ is symmetric about $\sigma_{I I}=0$.

When the stress state lies on the yield curve, irreversible plastic deformation occurs. The yield potential is identified with the plastic potential and an associated normal flow law is adopted,

$$
\dot{\varepsilon}_{k}^{p}=\left.\lambda \frac{\partial \mathcal{F}}{\partial \sigma_{k}}\right|_{\mathcal{F}=0}, \quad k=I, I I,
$$

where $\dot{\varepsilon}_{k}^{p}$ is the plastic strain rate and $\lambda$ is a positive scalar which is determined as part of the solution of the equations. This flow rule has been used in almost all models of sea ice dynamics. It has been successfully applied to granular materials such as soils, and gives satisfactory performance (within the limits of experimental error and parameter tuning) in sea ice simulations.

The viscous and rigid/plastic behavior can be represented using a nonlinearly viscous (or viscoplastic) model for an isotropic material,

$$
\sigma_{i j}=2 \eta \dot{\varepsilon}_{i j}+[\zeta-\eta] \dot{\varepsilon}_{k k} \delta_{i j}-P \delta_{i j} / 2,
$$

where $P$ is a pressure term, characteristic of the ice strength. The functions $\zeta\left(\dot{\varepsilon}_{i j} ; P\right)$ and $\eta\left(\dot{\varepsilon}_{i j} ; P\right)$ (traditionally referred to as bulk and shear viscosities in fluid dynamics) depend upon the strain rate (symmetric part of the velocity deformation tensor) $\dot{\varepsilon}_{i j}$ and $P$ so as to ensure that for typical strain rates the normal plastic flow law applies and the stress state lies on the yield curve. The shape of the yield curve is chosen to agree with the expected behavior of sea ice-that it should be weak in tension, strong in shear, and strongest in compression.

For almost 30 years since the VP rheology was introduced, work has focused on constraining the shape of the yield curve or creating numerically efficient algorithms for practical use. Most notable is the Elastic-Viscous-Plastic (EVP) rheology [HD97], which introduced an artificial elasticity that permits explicit numerical methods to be employed. The EVP rheology is used in many climate models today.

Work on constraining the shape of the yield curve and flow rule has followed two main approaches: homogenization or scale invariance. The homogenization approach attempts to calculate what would be the rheology of a homogenous material with the same aggregate properties as the unresolved (subgrid scale, $\approx 50 \mathrm{~km} \times 50 \mathrm{~km}$ ) region of heterogenous ice types and open water [S82]. Scale invariance asserts that the material rheology measured in the lab is fundamentally the same as the rheology of a grid-scale region and is of a Mohr-Coulombic character, e.g., [S107]. More details may be found in [Fel08].

Aside from the huge theoretical convenience of the assumption of isotropy, the most compelling argument cited in its favor is that on length scales of $100 \mathrm{~km}$ and greater, the distribution of leads appears to be nearly isotropic so that a mean-field rheology is isotropic. However, increasing evidence has shown that lead orientation, at least in the central pack, has a marked bimodality with the leads defining diamond-shaped floe aggregates, e.g., [S41,S86,S17]. While a number of approaches have been taken to simulate or parameterize the impact of the observed anisotropy of leads on sea ice rheology, there are currently three main strands of modeling. (i) The Elastic Decohesive model introduced to sea ice in [S85] explicitly simulates the formation of a crack or lead. (ii) The Elastic Brittle rheology, e.g., [S20], assumes an elastic subfailure response and a Coulombic failure criterion, with a new scalar damage parameter. When a grid cell fails, the elastic modulus is reduced, leading to local strain softening. Because of the long-range interactions within the elastic medium, local drops in the elastic modulus redistribute stress that can in turn induce damage. By this process, avalanches of damage events can occur, simulating the propagation of leads. (iii) The Elastic Anisotropic Plastic rheology also introduces a damage parameter, the anisotropic structure tensor, that gives the orientation of existing cracks within an element, and the mean stress is calculated as a function of crack orientation. In its most recent formulation [S108], the cracks are assumed to delineate diamond shaped floes/floe aggregates, motivated from observations.

As numerical resolution in sea ice models has increased, and as better observations of sea ice thickness and deformation become available from satellite imagery, e.g., CryoSat- 2 and IceSat-2, interest in fine scale simulation, e.g., $\approx 10 \mathrm{~km}$, of sea ice deformation has increased. This is helping motivate further work on developing realistic, and verifiable, models of sea ice rheology.

Large-scale numerical models. Most large-scale sea ice physical processes are reasonably well understood and represented in numerical climate models. For example, the first detailed thermodynamic description appeared almost 50 years ago [MU71]. Likewise, a relatively simple approach for sea ice dynamics is 40 years old [Hib79]. They were too computationally expensive to be incorporated in numerical models at the time, but have now been implemented in most GCMs. These thermodynamic and dynamic models capture the first-order behavior of sea ice in the climate system. Model development now follows two paths, both addressing higher-order effects: (1) more precise descriptions of key processes and characteristics such as microstructure evolution, anisotropy, and rheology, and (2) model extensions for "Earth system" simulations, e.g., by including biological and chemical species.

Modern sea ice models still use one-dimensional thermodynamic parameterizations, because the thermodynamic processes are still primarily vertical in nature. Most early sea ice models neglected thickness variations within 
a grid cell, but many models now include ice thickness distributions. As mentioned above, a floe size distribution is now being added to large-scale sea ice models, driven by thermodynamic growth and melting along with wave interactions.

In addition to the basic equations presented above for momentum, internal stress, and thermodynamics, numerical sea ice models also include detailed representations of radiative balance, surface characteristics and evolution (snow, snow-ice, melt ponds, albedo), ice strength, horizontal transport, and ridging. Some models now incorporate detailed descriptions of key players in the sea ice ecosystem, such as algae. These models must also interact with the other components of the Earth system, namely the atmosphere, ocean, and their ecosystems. These interactions are often tightly coupled. For instance, downwelling longwave radiation from clouds is a primary driver of sea ice surface temperature, which in turn determines upwelling longwave radiation, heating the overlying atmosphere and changing the cloud cover. Sea ice model development is best performed in a coupled modeling environment that includes such feedbacks.

Wind stress is arguably the primary forcing mechanism for the ice motion, although the ice-ocean stress, Coriolis force, and slope of the ocean surface are also important. Coupling between sea ice models and atmospheric models or data generally employs a quadratic form for the wind stress and for the ice-ocean stress term at the bottom of the ice. For ridging, modern models use an energy based description of mechanical redistribution that converts thinner ice to thicker ice under convergence and shear.

Sea ice albedo is critical for the global heat balance, and can be an effective "tuning knob" to produce a realistic simulation of sea ice extent. Simple, easily tunable albedo parameterizations specify four albedo values: cold snow; warm, melting snow; cold, bare ice; and warm, melting ice, while others use more complex formulations that take into account the ice and snow thickness, spectral band, and other parameters. Solar radiation may be distributed within the ice column assuming exponential decay (Beer's Law) or via multiple-scattering radiative transfer, in which absorptive effects of melt ponds and inclusions such as dust and algae can be simulated.

Sea ice is quite heterogeneous, mostly because of its salt content. In many coupled models, a fixed value of sea ice salinity is used at the ice-ocean interface, but internally the value can vary in time, or the model assumes a variable salinity profile that is constant in time. Newer thermodynamic approaches treat sea ice as a "mushy" layer of brine and ice [S24], parameterizing its desalination as it first grows and then transitions from first-year to multiyear ice. Prognostic representation of sea ice salinity and microstructure is critical for detailed sea ice ecosystem models, which depend on the permeability of the ice to allow flushing of the brine network by seawater, which carries nutrients into the ice and in turn seeds algal blooms in the ocean.

Melt water collects in depressions on the surface of the ice and can drain through brine channels when the ice becomes warm and permeable. By cleaning the ice of salt, nutrients, and other inclusions, this flushing mechanism can affect the albedo, conductivity, and biogeochemical processes and thereby play a role in climate change. The simplest pond scheme doesn't track melt water, but rather decreases ice surface albedo under warm, melting conditions. As discussed above, other methods track pond area and volume for each ice thickness category, to capture the radiative effect of melt ponds. More advanced pond schemes that simulate their hydrological influence, such as the delay of internal ice cooling as ponds refreeze in the fall, are under development.

Data assimilation. Sea ice models have developed to a degree that key features of the sea ice cover such as leads, ridges, and melt ponds, can be registered in the model output. As this model capacity evolves, we can hope to be able to predict with some accuracy where and when such features may occur. Nevertheless, the best numerical models may go astray after even possibly short periods of time. This model drift is mitigated by the incorporation of data from observations into the modeling process. This is typically done in two ways: (1) update the state of the system in model runs as observational data becomes available, and (2) learn parameter values from observational data. The first is known as state estimation and is carried out in real time, while the second process, called parameter estimation, is often achieved offline with the use of high resolution or more detailed models; both fit under the banner of Data Assimilation (DA) $\left[\mathrm{BBC}^{+} 17\right]$.

There are three main perspectives on DA, each having historical origin in one of: optimization, statistics, or control theory. The variational method balances observational data and model output through optimizing an appropriate cost function. In its time-dependent form, known as $4 \mathrm{D}$ Var, it is the basis of most schemes used in weather forecasting today. Nevertheless, most recent developments in DA methods have employed a statistical (Bayesian), a control theoretic (Kalman Filter based) approach, or a mixture of both. The key in these approaches is generating an ensemble of possible realizations of the process by considering slightly different initial conditions, parameters, or both. This ensemble of outputs is then used as a basis for a covariance in the Kalman Filter approach, or a prior probability distribution in a Bayesian method. Sea ice presents a number of interesting mathematical challenges to DA, with more complex models than those of the atmosphere and ocean because they account for ice 

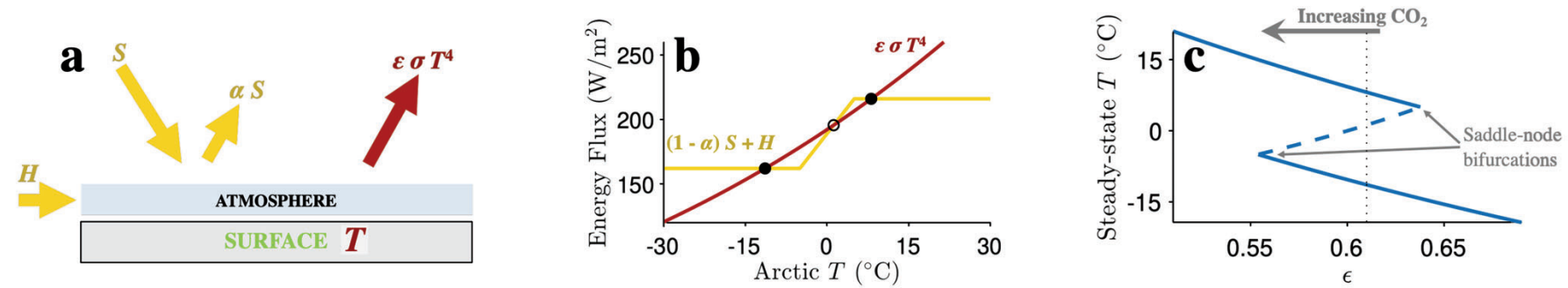

Figure 10. Simple model of how the ice-albedo feedback affects Arctic climate. (a) The main energy fluxes in the Arctic. (b) $E_{\text {in }}$ (yellow) and $E_{\text {out }}(\mathrm{red})$ in the energy balance as a function of $T$. Where both curves meet represent stable and unstable fixed points, which are indicated by filled and open circles, respectively. (c) Bifurcation diagram for the temperature as a function of the effective emissivity $\epsilon$. Increasing concentrations of atmospheric greenhouse gases cause a reduction in $\epsilon$.

material properties. The available data are both more varied and less direct. Synthetic-aperture radar data could potentially increase a model's fidelity to the location of features such as ridges and ponds.

Next generation models, such as neXtSIM [S78] and MPM-Ice [S98] have two novel features: they use rheologies not present in models currently incorporated into larger climate models, and more complicated computational solvers that include a Lagrangian aspect which affords a better accounting for the ice movement and the formation of localized features, and, at least in the case of neXtSIM, adaptive remeshing. Recent progress has been made on the development of a scheme tailored to adaptive remeshing [S5]. For any computational scheme, the formation of ridges and leads poses a challenge as current DA technology can break down in the presence of sharp transitions in space or time.

\section{Low-Order Models}

While large-scale numerical sea ice models have increased in complexity over time as more physical processes are accounted for, an alternative approach to studying sea ice and climate takes another path. This approach considers relatively simple mathematical models of key phenomena based on low-order dynamical systems and differential equations, that capture essential physics and provide insight into complex behavior, yet are more tractable than large numerical models.

Ice-albedo feedback. We consider a simple picture of the influence of the ice-albedo feedback, which can give rise to instability, and approximate the mean surface temperature $T$ of the Arctic region using a balance between incoming and outgoing energy fluxes, illustrated in Figure 10a. Energy balance can be written as $c \frac{d T}{d t}=E_{\text {in }}-E_{\text {out }}$, where $c$ is the effective heat capacity [S49]. Then

$$
c \frac{d T}{d t}=(1-\alpha) S+H-\epsilon \sigma T^{4},
$$

where $S=180 \mathrm{~W} / \mathrm{m}^{2}$ is the annual mean incident solar radiation in the Arctic region; $\alpha(T)$ is the albedo, modeled to have high reflectivity (0.6) for cold temperatures $T \leq-10^{\circ} \mathrm{C}$ and a lower value (0.3) for warmer temperatures $T \geq 10^{\circ} \mathrm{C}$, with linear interpolation in between; and $H=90 \mathrm{~W} / \mathrm{m}^{2}$ is the heat which enters the Arctic through poleward heat transport in the atmosphere. The Earth emits electromagnetic radiation to space with a radiant flux given by the Stefan-Boltzmann law, $\in \sigma T^{4}$, with $\sigma$ the Stefan-Boltzmann constant. Due to the greenhouse atmosphere of the Earth, the effective emissivity is $\epsilon=0.61$. $E_{\text {in }}$ and $E_{\text {out }}$ in equation (22) are plotted in Figure 10b, showing three possible steady-state solutions. One has a cold ice-covered Arctic, another has a warm ice-free Arctic. These two stable solutions are separated by an unstable one.

An increase in atmospheric greenhouse gas concentrations can be represented in this framework by reducing the effective emissivity $\epsilon$. Varying this causes the red line in Figure $10 \mathrm{~b}$ to be scaled vertically, leading to two saddle-node bifurcations which are shown in Figure 10c.

Variations of this simple idea have deep roots in climate science, going back more than a century [WE15, S68,S49], and there has been renewed interest in recent years due to the rapid Arctic sea ice retreat. However, a range of factors complicate the picture. First, there are spatial variations in the climate system, and the heat transport $(H)$ into the Arctic depends interactively on the spatial gradient in temperature. Energy balance models (EBMs) were developed a half century ago $[S 12, S 90]$ to explore such questions. They represent the annual zonal-mean surface temperature as a function of latitude under solar forcing, the ice-albedo feedback, and horizontal atmospheric heat transport via surface temperature diffusion. These models have multiple steady states, similar to Figure 10c (see [S68]).

Second, seasonal variations can be represented by varying $S$ in equation (22) over the course of the year. In this case the thermodynamics of sea ice growth and melt become relevant. Recent work used bifurcation theory to show that in a seasonally varying model that includes an idealized representation of the ice-albedo feedback, an 
unstable solution bracketed by saddle-node bifurcations occurs, similar to Figure 10c [EW09].

Recently, the effect of including both spatial variations and the seasonal cycle was explored using an idealized model that simulates the surface temperature and thickness of sea ice as a function of latitude and time [WE15]. The strength of the seasonal cycle and the horizontal heat transport are varied, and both factors have strong stabilizing effects. Including both causes the ice to always be stable in climates with the ice edge in midlatitudes or farther poleward, removing the bifurcations and instabilities previously encountered. Hence for Arctic sea ice covers resembling modern conditions and future sea ice reductions, this study found that no bifurcation should be expected to occur due to the ice-albedo feedback.

Sea ice concentration field. Low-order models can provide idealized sea ice concentration fields $\psi$ useful in data analysis applications. For a region $G$, the simplest model is perhaps Laplace's equation, $\Delta \psi=0$, using observations on $\partial G$ as Dirichlet boundary conditions. The idealized $\psi$ solves a steady-state heat equation, so is smoother than the actual field subjected to dynamic and thermodynamic forcing.
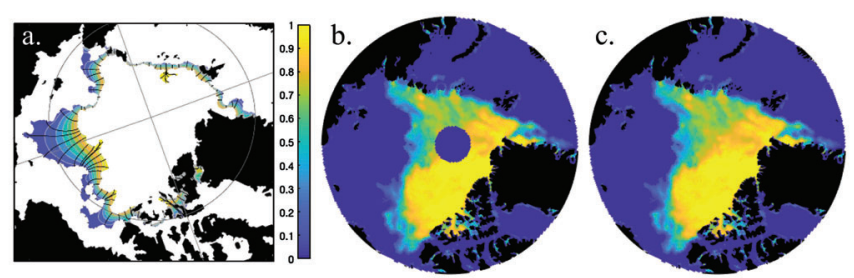

Figure 11. (a) Laplace equation solution in the MIZ for 29 August 2010 [S95]. (b,c) Filling the polar data gap with an idealized concentration field for 20 June 2013 [SG16].

As an example application, $\psi$ provides an idealized sea ice concentration field within the MIZ, which is the transition region between dense polar pack ice and the open ocean at lower latitudes (Figure 11a). The width of this highly dynamic zone is a fundamental length scale for polar physical and biological processes, and has increased $39 \%$ in the Arctic melt season over the past several decades [S96,S95]. The MIZ is in general not geodesically convex, but its width can be objectively defined as the arclength of streamlines through $\psi$ (black curves, Figure 11a). This arclength-based width is objective, unique at each point, and invariant with respect to rotation, translation, and coordinate system.

The idealized field $\psi$ has also been applied to fill in regions where satellite observations are missing or systematically unavailable, such as the polar data gap (Figure 11b). The fill is written

$$
f(\theta, \phi)=\psi(\theta, \phi)+w(\theta, \phi),
$$

where $\theta$ is longitude and $\phi$ is latitude, and $w$ is a stochastic term providing realistic spatial heterogeneity (Figure 11c). Boundary conditions for $\psi$ are sea ice concentrations observed on the boundary of the region being filled. The statistical properties of $w$ are determined from variations in the actual $\psi$ around the missing data region.

Conclusions and future challenges. The sea ice covers of the polar oceans are in transition and transitions provide challenges to modeling. A few decades ago the Arctic Ocean was predominantly ice covered throughout the summer. At present it is partially ice covered with large year to year variability in the amount, and the location, of summer ice. Model projections indicate that a few decades from now the Arctic Ocean will likely be predominantly ice free in summer. The receding ice cover has generated increased human activity that requires improvements in forecasts and modeling capabilities.

The complex multiscale nature of the sea ice system presents fundamental challenges in applied mathematics and computation. Homogenization theory and techniques of statistical physics for computing macroscopic behavior have been central to advancing mathematical modeling of sea ice. Given the stochastic nature of the sea ice system, sea ice models are often cast in a probabilistic framework, which also provides a robust set of tools to assimilate the vast amounts of data available from airand space-borne platforms, as well as large polar expeditions such as MOSAiC. Methods of dynamical systems and bifurcation theory have been successful in framing and analyzing qualitative questions about transitional behavior and pattern formation. Finally, substantial effort over the past 50 years has gone into, and continues to go into, developing large-scale numerical models that can predict sea ice behavior well into the future. Further development of the components of global climate models will need advances in high performance computing and learning from data, numerical techniques for solving large systems of coupled PDE, and mathematical methods to account for the microscale in macroscale behavior. 
ACKNOWLEDGMENTS. KMG would like to thank the US Office of Naval Research (ONR) and the US National Science Foundation (NSF) for their research support through grants N00014-18-1-2552, N00014-1310291, DMS-1715680, and DMS-1413454. LGB would like to thank the Australian Research Council and the Australian Antarctic Science Program for their research support through grants FT190100404, DP200102828, AAS4434, and AAS4528. EC would like to thank ONR and NSF for their research support through grants N00014-18-1-2552, DMS-1715680, and DMS1413454. IE would like to thank NSF for their support through grant OPP-1643445. CH thanks the Institute of Water and Atmospheric Science in New Zealand for their hospitality and acknowledges support from the Voss Postdoctoral Fellowship at Brown University and NASA Grant GR5227091. ECH acknowledges support through the Energy Exascale Earth System Model project within the Office of Biological and Environmental Research, Department of Energy. CJ was supported by ONR under grant N00014-18-1-2204. DKP would like to thank NSF and NOAA for their research support through grants NSF1724540 and NA19OAR4310094. The research of PPC has been supported by ONR under grant N00014-17-1-2076. CS would like to thank ONR and NASA for research support under grants N00014 18-1-2552, N00014-13-10291, and 80NSSC18K0843. AJW acknowledges support from the Royal Society International Exchanges Award IE141071. Finally, we also thank the reviewers for very helpful comments.

\section{References}

$\left[\mathrm{BBC}^{+}\right.$17] M. Buehner, L. Bertino, A. Caya, P. Heimbach, and G. Smith, Sea ice data assimilation, Sea ice analysis and forecasting: Towards an increased reliance on automated prediction systems, Cambridge University Press, Cambridge, 2017, pp. 9-164.

[DC19] S. Das and P. Ponte Castañeda, A multi-phase homogenization model for the viscoplastic response of intact sea ice: the effect of porosity and crystallographic texture., Int. J. Multiscale Comput. Eng. 17 (2019), 121-150.

[EW09] I. Eisenman and J. S. Wettlaufer, Nonlinear threshold behavior during the loss of Arctic sea ice, Proc. Natl. Acad. Sci. U.S.A. 106 (2009), no. 1, 28-32.

[Fel08] Daniel L. Feltham, Sea ice rheology, Annual review of fluid mechanics. Vol. 40, Annu. Rev. Fluid Mech., vol. 40, Annual Reviews, Palo Alto, CA, 2008, pp. 91-112, DOI 10.1146/annurev.fluid.40.111406.102151. MR2402388

[FSFH12] D. Flocco, D. Schroeder, D. L. Feltham, and E. C. Hunke, Impact of melt ponds on Arctic sea ice simulations from 1990 to 2007, J. Geophysical Research: Oceans 117 (2012), no. C9, C09032.
[Gol09] Kenneth M. Golden, Climate change and the mathematics of transport in sea ice, Notices Amer. Math. Soc. 56 (2009), no. 5, 562-584. MR2509061

[GAL98] K. M. Golden, S. F. Ackley, and V. I. Lytle, The percolation phase transition in sea ice, Science 282 (1998), 22382241.

[GLCG15] A. Gully, J. Lin, E. Cherkaev, and K. M. Golden, Bounds on the complex permittivity of polycrystalline materials by analytic continuation, Proc. Roy. Soc. A. 471 (2015), no. 2174, 20140702, 17, DOI 10.1098/rspa.2014.0702 MR3328346

[Hib79] W. D. Hibler, A dynamic thermodynamic sea ice model, J. Phys. Oceangr. 9 (1979), no. 4, 815-846.

[HT15] C. Horvat and E. Tziperman, A prognostic model of the sea-ice floe size and thickness distribution, The Cryosphere 9 (2015), no. 6, 2119-2134.

[HD97] E. C. Hunke and J. K. Dukowicz, An elastic-viscousplastic model for sea ice dynamics, J. Phys. Oceanogr. 27 (1997), 1849-1867.

[MSSG19] Yi-Ping Ma, Ivan Sudakov, Courtenay Strong, and Kenneth M. Golden, Ising model for melt ponds on Arctic sea ice, New J. Phys. 21 (2019), no. June, 063029, 8, DOI 10.1088/1367-2630/ab26db. MR3979993

[MU71] G. A. Maykut and N. Untersteiner, Some results from a time dependent thermodynamic model of sea ice, J. Geophys. Res. 76 (1971), no. 6, 1550-1575.

[MSB16] Fabien Montiel, V. A. Squire, and L. G. Bennetts, Attenuation and directional spreading of ocean wave spectra in the marginal ice zone, J. Fluid Mech. 790 (2016), 492-522, DOI 10.1017/jfm.2016.21 MR3482130

[PMWK20] James R. G. Parkinson, Daniel F. Martin, Andrew J. Wells, and Richard F. Katz, Modelling binary alloy solidification with adaptive mesh refinement, J. Comput. Phys. X 5 (2020), 100043, DOI 10.1016/i.jcpx.2019.100043 MR4116091

[SFFT14] D. Schroeder, D. L. Feltham, D. Flocco, and M. Tsamados, September Arctic sea-ice minimum predicted by spring melt-pond fraction, Nat. Climate Ch. 4 (2014), 353357.

[SG16] C. Strong and K. M. Golden, Filling the polar data gap in sea ice concentration fields using partial differential equations, Remote Sensing 8 (2016), no. 6, 442-451.

[TRMC75] A.S. Thorndike, D. A. Rothrock, G. A. Maykut, and R. Colony, The thickness distribution of sea ice, J. Geophys. Res. 80 (1975), no. 33, 4501-4513.

[WE15] T. J. W. Wagner and I. Eisenman, How climate model complexity influences sea ice stability, J. Climate 28 (2015), no. $10,3998-4014$

[WHP19] A. J. Wells, J. R. Hitchen, and J. R. G. Parkinson, Mushy-layer growth and convection, with application to sea ice, Phil. Trans. Royal Soc. A: Mathematical, Physical and Engineering Sciences 377 (2019), no. 2146, 20180165.

Supplemental reference list located here www. math.utah . edu/ go/den/docs/publications/Golden_NAMS _Supplemental_Reterences_Nov_2020.pd† 


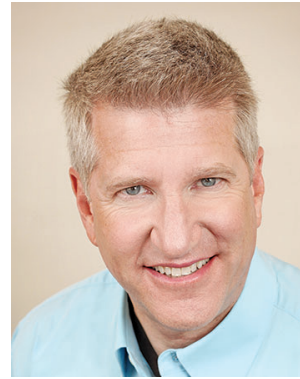

Kenneth M. Golden

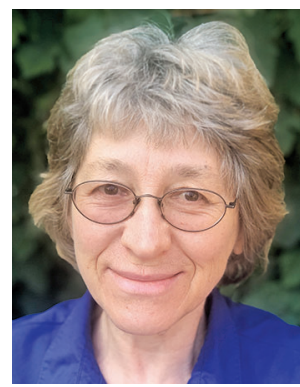

Elena Cherkaev

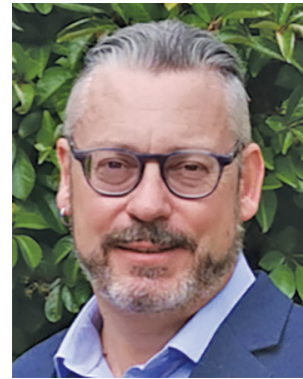

Daniel Feltham

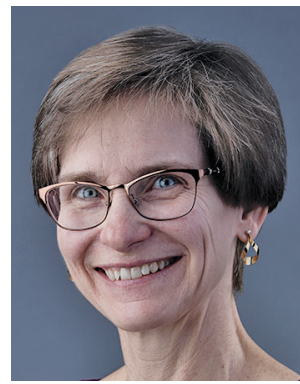

Elizabeth Hunke

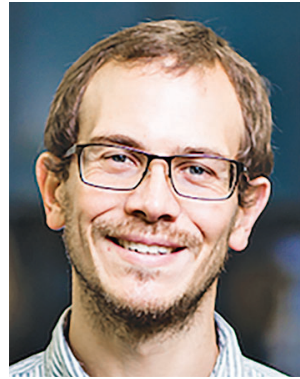

Luke G. Bennetts

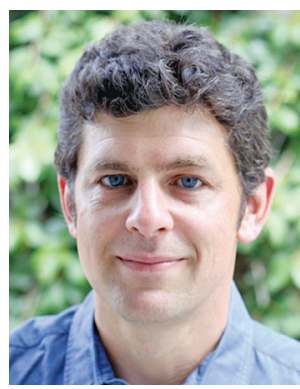

Ian Eisenman

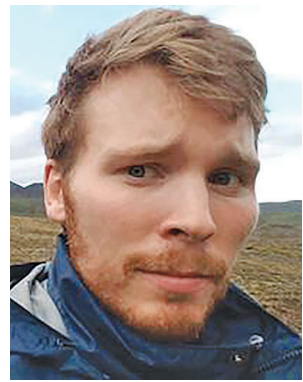

Christopher Horvat

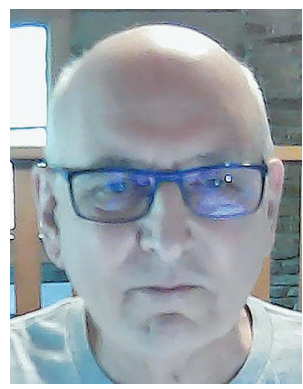

Christopher Jones

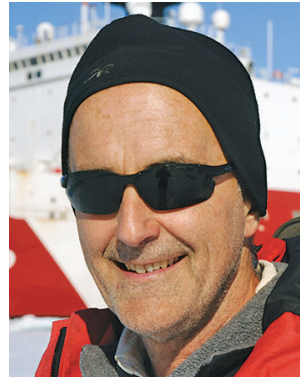

Donald K. Perovich

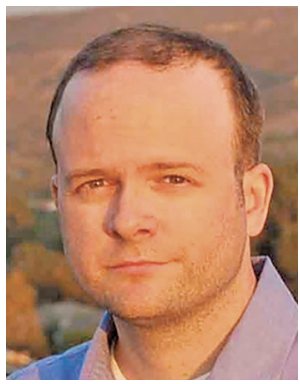

Courtenay Strong

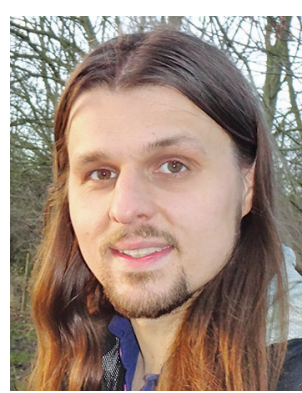

Andrew J. Wells

Credits

Opening graphic is courtesy of A. Toffoli from the S. A. Agulhas II.

Figure 1 is courtesy of, from left to right, Hajo Eicken and Kenneth M. Golden, Pat Langhorne and Kenneth M. Golden, Donald Perovich, Donald Perovich, NASA Worldview.

Figure 2 is courtesy of Samuel Torquato ( $a$ and b) and the authors (c).

Figure 3 is courtesy of Pat Langhorne (left) and Jean-Louis Tison (right).

Figures 4, 5, 10, and 11 are courtesy of the authors.

Figure 6 is courtesy of Kenneth M. Golden.

Figure 7 is courtesy of Takenobu Toyota.

Figure 8 is courtesy of Donald K. Perovich.

Figure 9 is courtesy of Kenneth M. Golden (on behalf of Yiping Ma, Ivan Sudakov, and Courtenay Strong) (left) and Donald K. Perovich (right).

Author photo of Kenneth M. Golden is courtesy of Todd Collins.

Author photo of Luke G. Bennetts is courtesy of Randy Lacombe. 
Author photo of Elena Cherkaev is courtesy of Andrej Cherkaev.

Author photo of Ian Eisenman is courtesy of Ian Eisenman.

Author photo of Daniel Feltham is courtesy of Malcolm Edwards.

Author photo of Christopher Horvat is courtesy of Christopher Horvat.

Author photo of Elizabeth Hunke is courtesy of Elizabeth Hunke/Los Alamos National Laboratory Media Services.

Author photo of Christopher Jones is courtesy of Christopher Jones.

Author photo of Donald K. Perovich is courtesy of Kathryn Hansen.

Author photo of Pedro Ponte-Castañeda is courtesy of the School of Engineering and Applied Sciences, University of Pennsylvania.

Author photo of Courtenay Strong is courtesy of Natasha Strong.

Author photo of Deborah Sulsky is courtesy of Deborah Sulsky.

Author photo of Andrew J. Wells is courtesy of Andrew J. Wells.

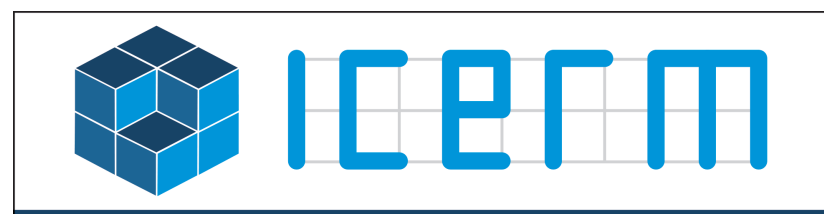

Institute for Computational and Experimental Research in Mathematics

OPEN POSITIONS:

Deputy Director \& Postdocs

ICERM Deputy Director: ICERM has a half-time opening for a Deputy Director starting in summer 2021. We are looking for someone with a Ph.D. and research achievements in mathematics or a related field who can assist our Director in soliciting, developing, and overseeing our scientific programs. Applicants should submit a cover letter, CV, and names of two references to director@icerm.brown.edu.Review of applications will begin November 15, 2020.

ICERM Postdoctoral Fellowships: These semester and institute positions are affiliated with ICERM's 2021-2022 semester programs - fall 2021 "Hamiltonian Methods in Dispersive and Wave Evolution Equations" and spring 2022 "Braids". These four and nine-month positions come with salary and benefits and are matched with a faculty mentor.

Simons Bridge Postdoctoral Fellowships: In response to the COVID-19 crisis, the Simons Foundation has created the Simons Bridge for Postdoctoral Fellowships. These positions provide additional funding to institutes like ICERM to support one-year postdoctoral positions in mathematics starting in the fall of 2021 and 2022. These are twelve-month appointments, and may be extendable for a second year.

Details for all of these positions can be found at MathJobs.org: http://mathjobs.org/jobs/ICERM

Brown University is committed to fostering a diverse and inclusive academic global community; as an EEO/AA employer, Brown considers applicants for employment without regard to, and does not discriminate on the basis of, gender, sex, sexual orientation, gender identity, national origin, age, race, protected veteran status, disability, or any other legally protected status.

Ways to participate:

Propose a:

- semester program

- topical workshop

- small group research program

- summer undergrad program

Apply for a:

- semester program or workshop

- postdoctoral fellowship

Become an:

- academic or corporate sponsor
About ICERM: The Institute for Computational and Experimental Research in Mathematics is a National Science Foundation Mathematics Institute at Brown University in Providence, RI.

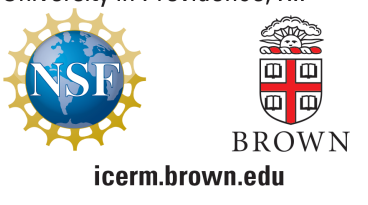

